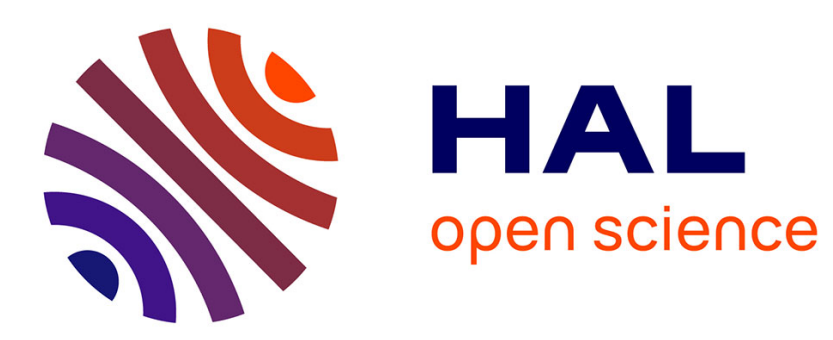

\title{
Données récentes sur les habitats ruraux du premier âge du Fer en Centre-Ouest
}

Christophe Maitay

\section{To cite this version:}

Christophe Maitay. Données récentes sur les habitats ruraux du premier âge du Fer en Centre-Ouest. Documents d'archéologie méridionale, 2015, 36, pp.353-372. hal-02265014

\section{HAL Id: hal-02265014 \\ https://hal.science/hal-02265014}

Submitted on 8 Aug 2019

HAL is a multi-disciplinary open access archive for the deposit and dissemination of scientific research documents, whether they are published or not. The documents may come from teaching and research institutions in France or abroad, or from public or private research centers.
L'archive ouverte pluridisciplinaire $\mathbf{H A L}$, est destinée au dépôt et à la diffusion de documents scientifiques de niveau recherche, publiés ou non, émanant des établissements d'enseignement et de recherche français ou étrangers, des laboratoires publics ou privés. 
Virginie ROPIOT, Florent MAZIÈRE

Introduction

\section{Présentations monographiques et micro-régionales}

- Florent MAZIÈRE, Céline JANDOT, Stéphanie RAUX, Marie-Pierre RUAS, Vianney FOREST, Antoine RATSIMBA, Pascal VERDIN, Jérôme ROS, Anne BORVON. . . . . . . . . . . . . . . . . . . . . . 19 Une aire d'ensilage du deuxième âge du Fer en Roussillon : les Vignes de l'Espérance (Banyuls-dels-Aspres, Pyrénées-Orientales)

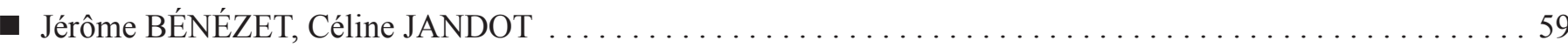
La fosse du deuxième âge du Fer des Estanyols (Pia, Pyrénées-Orientales)

- Aurélien ALCANTARA, Julien BOHNY, Géraldine CAMAGNE, Camille FAÏSSE, Florent RUZZU . . . . . 73 Note préliminaire sur l'habitat de Quinquiris (Castelnaudary, Aude) à la fin du premier âge du Fer

- Christophe RANCHÉ

Les établissements ruraux de l'âge du Fer dans l'ouest du département de l'Aude : remarques préliminaires

- Virginie ROPIOT avec la collaboration de Katrina ANKER et Alexa STEVENSON $\ldots \ldots \ldots \ldots \ldots \ldots \ldots$

Un hameau du Ve s. av. J.-C. en Minervois: la Condamine (Villeneuve-Minervois, Aude)

- Florent MAZIÈRE, Benoit SENDRA, Vianney FOREST, Isabel FIGUEIRAL, Mélanie PRUVOST. . . 131 Les Garennes (Tourbes, Hérault): une aire d'ensilage du premier âge du Fer

- Pierre RASCALOU, Vianney FOREST, Isabel FIGUEIRAL, Claire-Anne DE CHAZELLES, Philippe ECARD . . . 155 L'Arnoux (Saint-Félix-de-Lodez, Hérault): un habitat rural du III ${ }^{\mathrm{e}}$ s. av. J.-C. de la moyenne vallée de l'Hérault

- Antoine RATSIMBA, Lise DAMOTTE, Vianney FOREST, Sophie MARTIN avec la collaboration de Pascale CHEVILLOT.

Mas Vigier (Nîmes, Gard), un exemple d'occupation en plaine nîmoise (seconde moitié du $\mathrm{V}^{\mathrm{e}} \mathrm{s}$. / premier quart du $\mathrm{IV}^{\mathrm{e}} \mathrm{s}$. av. n. è.)

- Pierre SÉJALON, Philippe CAYN, Nathalie CHARDENON, Isabel FIGUEIRAL, Vianney FOREST

Aménagements et évolution de l'exploitation agricole du Mas de Vignoles IX entre la fin $\mathrm{du} \mathrm{VI}^{\mathrm{e}}$ et le $\mathrm{IV}^{\mathrm{e}} \mathrm{s}$. av. n. è. à Nîmes (Gard)

- Alexandrine LE ROUZÈS, Hervé PETITOT

Résultats de prospections pédestres autour de l'oppidum Saint-Vincent (Gaujac, Gard)

\section{Approches thématiques}

- Isabel FIGUEIRAL, Laurent BOUBY, Lucie CHABAL, Charlotte HALLAVANT, Carmen MACHADO, Jérôme ROS, Marie-Pierre RUAS, Caroline SCHAAL, Véronique ZECH-MATTERNE . . . . . . . . . . 285 Données archéobotaniques sur les établissements ruraux du second âge du Fer en Languedoc-Roussillon

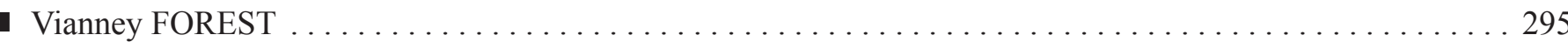
Données archéozoologiques sur les sites ruraux du Bas-Languedoc entre le $\mathrm{VI}^{\mathrm{e}}$ et le $\mathrm{III}^{\mathrm{e}} \mathrm{s}$. av. J.-C. : première synthèse 


\section{Contributions extra-régionales}

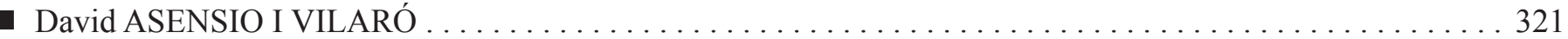

El poblament rural en les comunitats ibèriques del nord-est de la Península Ibèrica entre els segles VI i III aC.

- Philippe GARDES, Jean-Jacques GRIZEAUD ...

Le Toulousain et ses marges : recherches récentes sur les habitats ( $\mathrm{VI}^{\mathrm{e}}-\mathrm{III}^{\mathrm{e}} \mathrm{s}$. av. J.-C.)

- Christophe MAITAY .

Données récentes sur les habitats ruraux du premier âge du Fer en Centre-Ouest 


\section{Données récentes sur les habitats ruraux du premier âge du Fer en Centre-Ouest}

\section{Résumé :}

Cette contribution propose de faire le point sur les modalités d'occupation des campagnes du centre-ouest de la France entre le IX et le IV ${ }^{e}$ s. av. J.-C. Le développement de l'archéologie préventive permet depuis quelques années de préciser la question de l'occupation des campagnes au cours de la première moitié du premier millénaire avant notre ère. La multiplication des opérations et l'opportunité d'intervenir sur de grandes surfaces favorisent aujourd'hui les possibilités de saisir non seulement l'organisation des établissements ruraux du premier âge du Fer, mais également de discuter de leurs productions et de leurs interactions avec le milieu naturel. En Poitou-Charentes, les modes architecturaux s'inspirent encore de ceux de l’âge du Bronze, mais quelques spécificités, notamment la construction d'enclos palissadés, semblent se dégager. D'un point de vue spatial, les résultats attestent d'un réseau de sites beaucoup plus dense, annonçant peut-être ainsi le maillage mis en place par les populations gauloises à partir de La Tène moyenne.

Mots-clés :

Habitat rural, enclos, palissade, structure de stockage, centre-ouest de la France, Poitou-Charentes, premier âge du Fer.

\section{Abstract:}

This paper aims at reviewing the situation of west-central France countrysides occupation between the ninth and the fourth century BC. The development of rescue archaeology has only recently enabled us to clarify the question of the countryside occupation during the first half of the first millenium BC. Nowadays, the multiplication of operations and the opportunity to intervene on large surfaces favour many possibilities not only to understand the organisation of the Early Iron Age rural settlements, but also to discuss their productions and interactions with their natural environment. In Poitou and Charentes, the architectural patterns are still based on those of the Bronze Age, but some new special features (including the construction of palisade enclosures) seem to be emerging. From a spatial point of view, the results show a much denser network of sites which may be the sign of the network established by the Gaulish populations since the middle La Tène. 


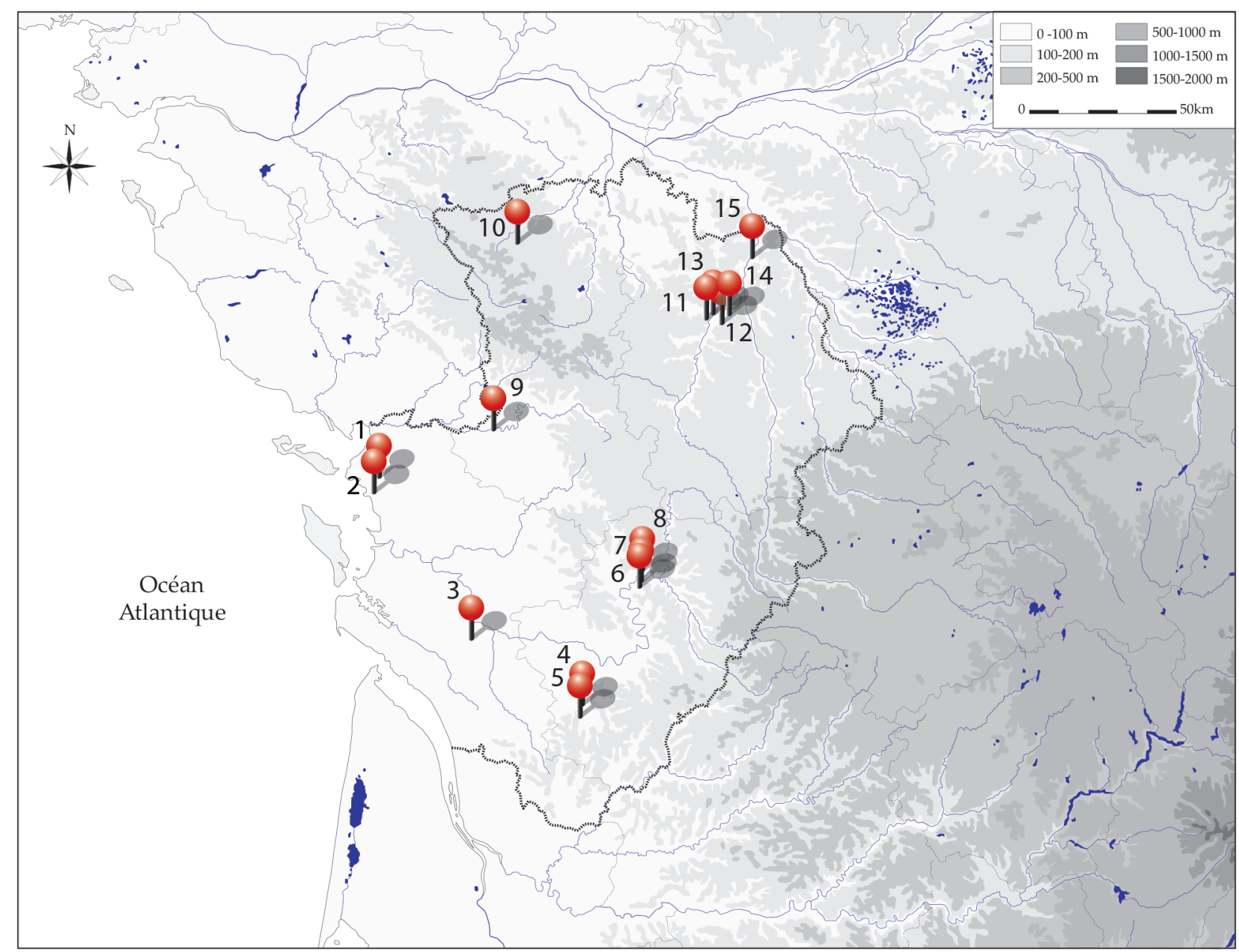

1 Localisation des sites mentionnés dans le texte (DAO C. Maitay). 1 : Les Drouillards à Dompierre-sur-Mer, Charente-Maritime ; 2 : Les Ormeaux à Angoulins, Charente-Maritime ; 3 : Le Pédeau à Préguillac, Charente-Maritime ; 4 : Les Petits Clairons II à Barbezieux, Charente ; 5 : La Fontaine 1 à Reignac-Barbezieux, Charente ; 6 : La Brangerie à Villognon, Charente ; 7 : Les Fioux à Villognon, Charente ; 8 : Le Mas de Champ Redon à Luxé, Charente ; 9 : Le Coteau de Montigné à Coulon, Deux-Sèvres ; 10 : Bois Roux à SaintAubin-du-Plain, Deux-Sèvres ; 11 : Malaguet à Migné-Auxances, Vienne ; 12 : Terre-qui-Fume à Buxerolles, Vienne ; 13 : Les Grands Philambins à Chasseneuil-du-Poitou, Vienne ; 14 : Les Gains à Saint-Georges-Lès-Baillargeaux, Vienne ; 15 : Les Terres Rouges à Ingrandes-sur-Vienne, Vienne.

\section{Introduction}

Le développement de l'archéologie préventive a largement favorisé le renouvellement de l'information sur les populations rurales protohistoriques et sur la structuration de leurs terroirs (entre autres Bertrand et al. 2009 ; Séjalon et al. 2009 ; Brun, Marcigny 2012). Si les habitats des campagnes du second âge du Fer ont bénéficié depuis les années 1970 d'un réel engouement de la part des archéologues, la période comprise entre le Bronze final IIIb et La Tène ancienne est restée longtemps à l'écart des programmes de recherche collectifs et des synthèses régionales (Maitay, Nillesse 2014). Dans les pays du Centre-Ouest (Poitou, Charentes et
Limousin), l'attention des protohistoriens, longtemps focalisée sur les établissements ruraux laténiens (Bertrand et al. 2009 ; Maguer, Lusson 2009), s'est trop rarement portée sur l'occupation du sol entre le IX ${ }^{\mathrm{e}}$ et le IV $\mathrm{IV}^{\mathrm{e}}$ s. av. J.-C. La plupart des découvertes se limite en effet bien souvent à des structures isolées (fosses et trous de poteau), entrevues à l'occasion de diagnostics ou de fouilles de faibles superficies. Face à l'extrême pauvreté des contextes funéraires du premier âge du Fer, les données sur l'occupation du sol et l'évolution des cultures matérielles sont encore dépendantes des travaux effectués depuis la fin du XIX ${ }^{\mathrm{e}}$ siècle sur les habitats groupés fortifiés (Maitay et al. 2009b).

L'objectif de cette contribution est de présenter quelques données récentes sur l'occupation des campagnes au pre- 
mier âge du Fer dans la région Poitou-Charentes. Il s'agit notamment, à partir d'un corpus d'une quinzaine de sites occupant des positions géographiques et topographiques variées, d'engager une réflexion sur la morphologie des habitats dispersés, l'architecture des bâtiments domestiques et agricoles, ou les modalités de stockage des récoltes.

\section{Les sources archéologiques}

Les occupations retenues ici sont exclusivement localisées en Poitou-Charentes, région constituée de plateaux calcaire de faible altitude parfois entaillés de profondes vallées (fig. 1). Elles se répartissent principalement près de voies navigables (Malaguet à Migné-Auxances, les Grands Philambins à Chasseneuil-du-Poitou, Terre-quiFume à Buxerolles, les Gains à Saint-Georges-Lès-Baillargeaux au bord de la vallée du Clain ; les Terres Rouges à Ingrandes-sur-Vienne, au bord de la Vienne ; la Brangerie et les Fioux à Villognon, le Mas de Champ Redon à Luxé, près du cours de la Charente ; le Coteau de Montigné à Coulon, au bord de la Sèvre niortaise) et le long du littoral charentais (les Ormeaux à Angoulins et les Drouillards à Dompierre-sur-Mer, en Charente-Maritime). Elles occupent des positions topographiques variées mais les plateaux calcaires et les versants sont les mieux représentés (les Petits Clairons II à Barbezieux, la Fontaine 1 à Reignac, Champ Redon et les Fioux à Luxé, la Brangerie à Villognon, en Charente; le Pédeau à Préguillac, en Charente-Maritime ; le Coteau de Montigné à Coulon, dans les Deux-Sèvres ; Terre-qui-Fume à Buxerolles, les Grands Philambins à Chasseneuil-du-Poitou, les Gains à Saint-Georges-Lès-Baillargeaux, dans la Vienne). Les plateaux calcaires littoraux sont également occupés (les Ormeaux à Angoulins et les Drouillards à Dompierresur-Mer, en Charente-Maritime), de même que les plaines alluviales, beaucoup plus fertiles et bénéficiant de conditions de conservation plus favorables ${ }^{1}$ (les Terres Rouges à Ingrandes-sur-Vienne, Malaguet à Migné-Auxances, dans la Vienne).

Quinze occupations, ayant fait l'objet de fouilles programmées et préventives ou de diagnostics, ont été retenues (tab. 1). Ces gisements sont occupés entre le IX et le $\mathrm{V}^{\mathrm{e}}$ s. av. J.-C., soit entre le Bronze final IIIb et le début de La Tène ancienne. À titre de comparaison, quelques sites de La Tène A2-B1 ont toutefois été inclus au corpus. Les données, recueillies entre 1978 et 2012, proviennent, à l'exception des fouilles du Coteau de Montigné à Coulon dans les Deux Sèvres, de fouilles préventives réalisées par l'AFAN puis l'INRAP et, dans une moindre mesure, par des opérateurs privés.

Précisons que plus de la moitié de ces sites ont été étudiés sur des surfaces inférieures à un hectare (tab. 1). Cette constatation restreint nécessairement les possibilités de

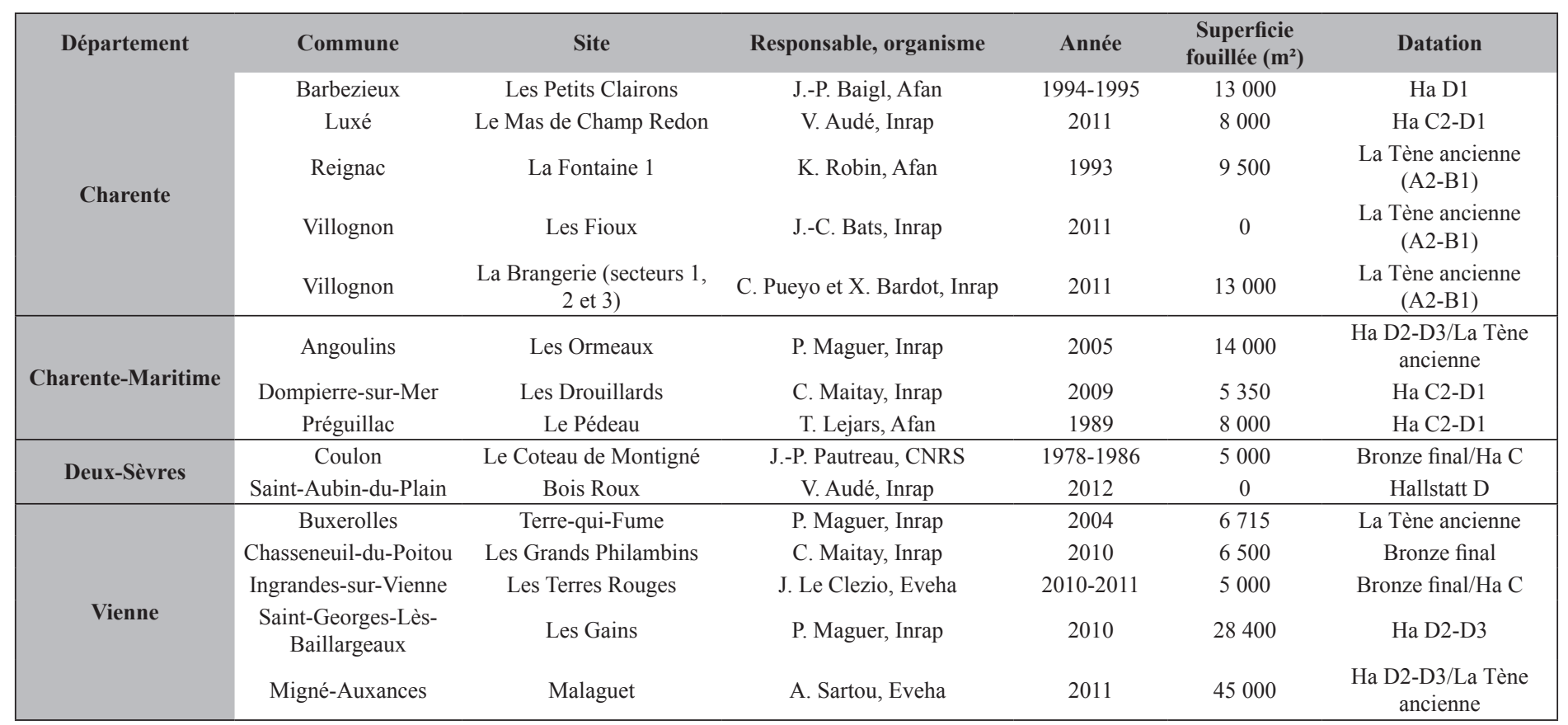

- Tableau 1 Inventaire des sites retenus pour cette étude. 
saisir à la fois l'ampleur de l'occupation et les relations avec son terroir, notamment avec d'éventuelles structures parcellaires ou funéraires. Si les superficies fouillées varient généralement entre 5000 et $8000 \mathrm{~m}^{2}$, cinq sites ont pu faire l'objet de décapages plus importants : les Petits Clairons II à Barbezieux (1,3 ha), les différents secteurs de la Brangerie qui, cumulés, atteignent une superficie de 1,3 ha, les Ormeaux à Angoulins (1,4 ha), les Gains à Saint-Georges-Lès-Baillargeaux ( $2,8 \mathrm{ha})$ et le site de Malaguet à Migné-Auxances (4,5 ha).

\section{Des établissements ruraux palissadés}

\subsection{Les enclos palissadés}

Plusieurs opérations de fouille récemment menées sur des habitats ruraux picto-charentais révèlent l'implantation de ces derniers à l'intérieur d'enclos de plan quadran-

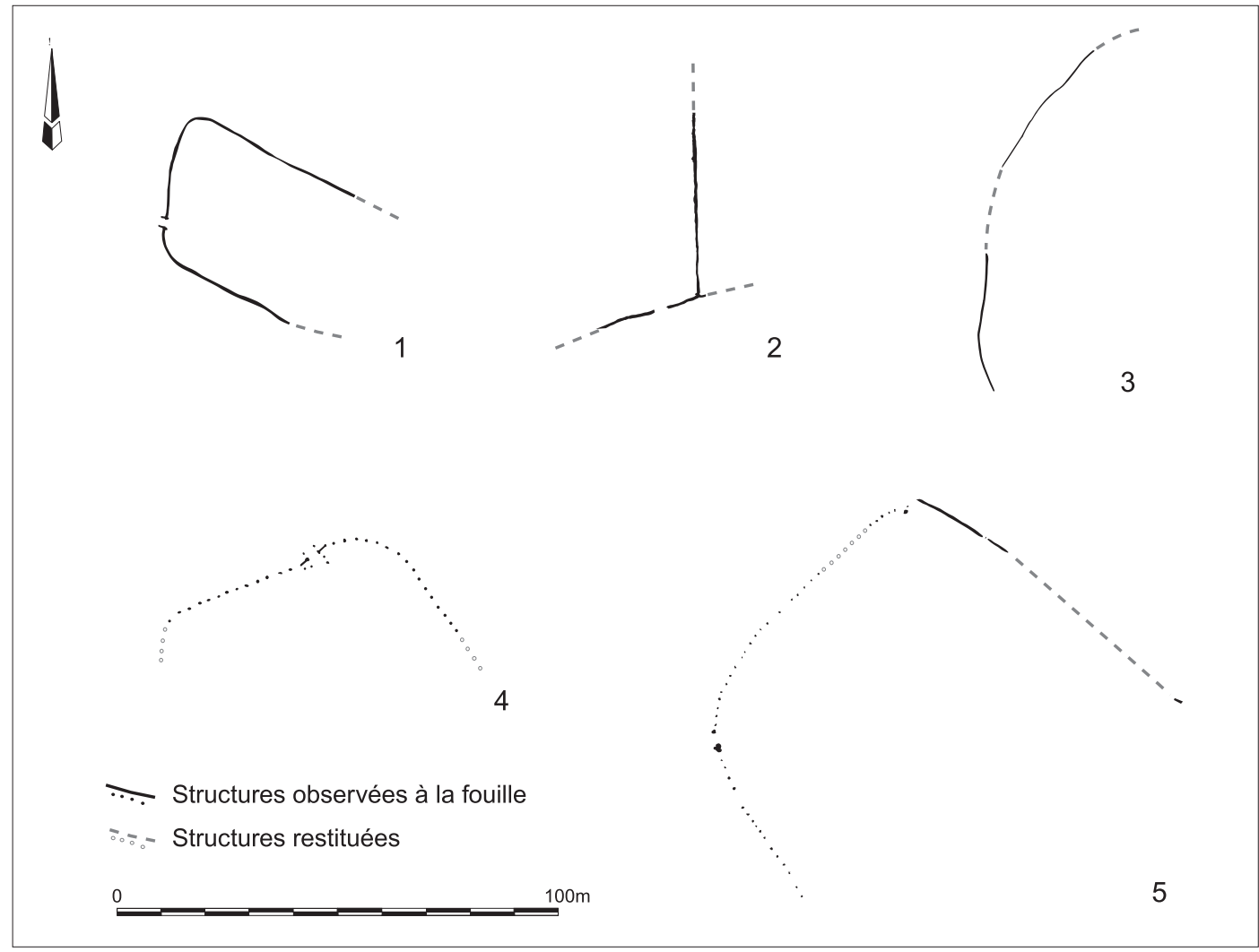

12 Enclos palissadés du premier âge du Fer (DAO C. Maitay). 1 : Les Drouillards à Dompierre-surMer, Charente-Maritime (d'après Maitay 2012) ; 2 : Le Coteau de Montigné à Coulon, Deux-Sèvres (d'après Pautreau 1981 et 1983) ; 3 : Les Petits Clairons II à Barbezieux, Charente (d'après Baigl et al. 1999) ; 4 : Le Pédeau à Préguillac, Charente-Maritime (d'après Lejars et al. 1990) ; 5 : Les Fioux à Villognon, Charente (d'après Bats dir. 2011). gulaire (fig. 2, $\mathrm{n}^{\text {os }} 1,2,4$ et 5). La fouille de l'habitat des Petits Clairons II à Barbezieux (Baigl et al. 1999), dont l'emprise adopte un tracé linéaire qui ne permet pas de définir l'intégralité de l'occupation, pourrait quant à elle démontrer le recours à une palissade de forme circulaire ou du moins en partie curviligne (fig. $2, \mathrm{n}^{\circ} 3$ et fig. 3 ). L'absence d'intervention ayant permis la fouille intégrale d'habitats de ce type empêche pour l'instant de proposer une classification détaillée de ces enclos ou de discuter de leurs statuts, comme cela a été effectué, par exemple, en Champagne (Desbrosses, Riquier 2012).

Dans tous les cas, l'enclos est constitué d'une palissade en matériaux périssables. L'existence de fossés périphériques, à la manière des établissements ruraux gaulois de l'ouest de la France, n'est pour l'instant pas attestée entre le premier âge du Fer et La Tène ancienne (Maguer, Lusson 2009, 427-431). Le plan rectangulaire ou trapézoïdal à angles arrondis est le plus fréquent (les Drouillards, le Pédeau, les Fioux), mais n'est pas le seul recensé. D’autres occupations, qui s'étendent sur des superficies conséquentes (Malaguet à Migné-Auxances, Vienne : Sartou, Delalande et al. 2012), n'ont pas livré pour autant de restes d'enclos clairement identifiés : elles pourraient correspondre à des habitats dispersés ouverts.

Deux principaux types de clôture peuvent être néanmoins définis : celui sur tranchée de fondation et celui sur file de poteaux. Le premier s'appuie sur une tranchée aménagée dans la roche. C'est par exemple le cas aux Petits Clairons II (fig. 2, n 3 ), en Charente, et aux Drouillards (fig. 2, $\mathrm{n}^{\mathrm{o}} 1$ ), en Charente-Maritime. Sur ce site (fig. 4), la zone d'habitat est fermée par une tranchée étroite, creusée dans le calcaire, et mesurant $0,45 \mathrm{~m}$ de largeur moyenne pour une profondeur conservée de $0,30 \mathrm{~m}$ (fig. $5, \mathrm{n}^{\text {os }} 1$ et 2 ) (Maitay 2012). Cet aménagement accueille à intervalles plus ou moins réguliers des 
poteaux de bois circulaires, visiblement non équarris (chêne sessile, chêne rouvre et chêne pubescent), d'une vingtaine de centimètres de diamètre au maximum, parfois calés par des blocs de pierre (fig. $5, n^{\circ} 3$ ). Ces poteaux supportaient un clayonnage de branches et de branchages dont les restes carbonisés ont été découverts dans les portions de tranchées entre deux supports verticaux. L'enclos, d'une largeur de $32 \mathrm{~m}$ pour une longueur totale inconnue, adopte une forme trapézoïdale régulière aux angles arrondis. Sa superficie estimée est, comme ses homologues du Pédeau et des Fioux, d'environ $5000 \mathrm{~m}^{2}$. La partie orientale ne semble pas construite, ce qui pourrait révéler comme au Coteau de Montigné (Pautreau 1981 ; 1983) un espace ouvert. La partie sud du segment occidental de l'enclos est interrompue par une entrée ouvrant vers le nord-ouest, orientée en direction d'un second accès, de forme identique mais dissocié de tout aménagement de clôture (fig. 4). Il n'existe apparemment pas sur ce site de système de partition interne, comme cela est connu sur d'autres établissements contemporains. Aux Petits Clairons II, une tranchée curviligne de 0,20 à $0,30 \mathrm{~m}$ de largeur et 0,30 à $0,40 \mathrm{~m}$ de profondeur conservée a pu être observée sur environ $55 \mathrm{~m}$ (fig. $2, \mathrm{n}^{\circ} 3$ ). L'hypothèse d'une palissade clôturant l'habitat est envisageable, mais il pourrait également s'agir d'une partition interne destinée à séparer deux secteurs de l'occupation.

Les établissements ruraux des Fioux (fig. 6) et du Pédeau (fig. 7) correspondent pour leur part au second type (Bats, Pueyo 2011 ; Lejars et al. 1990). Ils disposent d'un enclos constitué de poteaux de bois régulièrement implantés dans le substrat calcaire (fig. 2, $\mathrm{n}^{\text {os }} 4$ et 5). Ces poteaux prennent position dans des creusements à fond plat et paroi verticale : ils sont espacés les uns des autres de $2 \mathrm{~m}$ à Villognon et de 2,50 m à Préguillac. Au Pédeau, l'enclos est constitué de trous de poteau circulaires de 0,50 à $0,60 \mathrm{~m}$ de diamètre et 0,20 à $0,30 \mathrm{~m}$ de profondeur conservée.
Celui des Fioux, malheureusement non fouillé et aujourd'hui inaccessible, permet de définir une variante mixte mettant en œuvre une file de poteaux observée sur environ $140 \mathrm{~m}$ (côtés sud et ouest) et un petit fossé correspondant probablement à une tranchée de palissade (côté nord) (fig. 6). Les poteaux prennent place dans des creusements circulaires de 0,60 à $0,80 \mathrm{~m}$ de diamètre et de 0,20 à $0,40 \mathrm{~m}$ de profondeur conservée (Bats, Pueyo 2011, 36-38). Aucune structure suggérant l'existence d'un

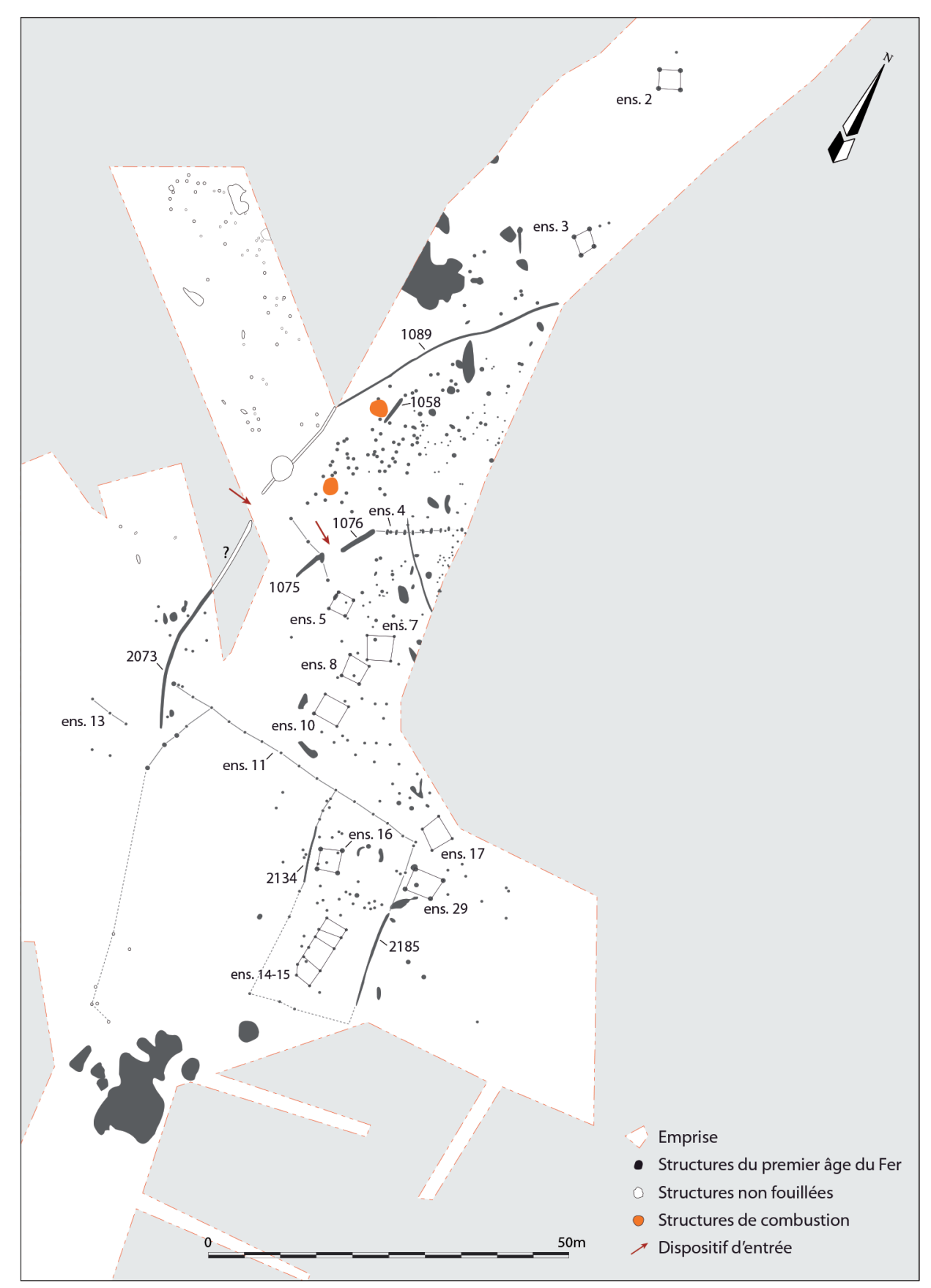

I 3 Les Petits Clairons II à Barbezieux, Charente. Plan de l'habitat du premier âge du Fer (DAO C. Maitay, d'après Baigl et al. 1999, fig. 3 et 4). 


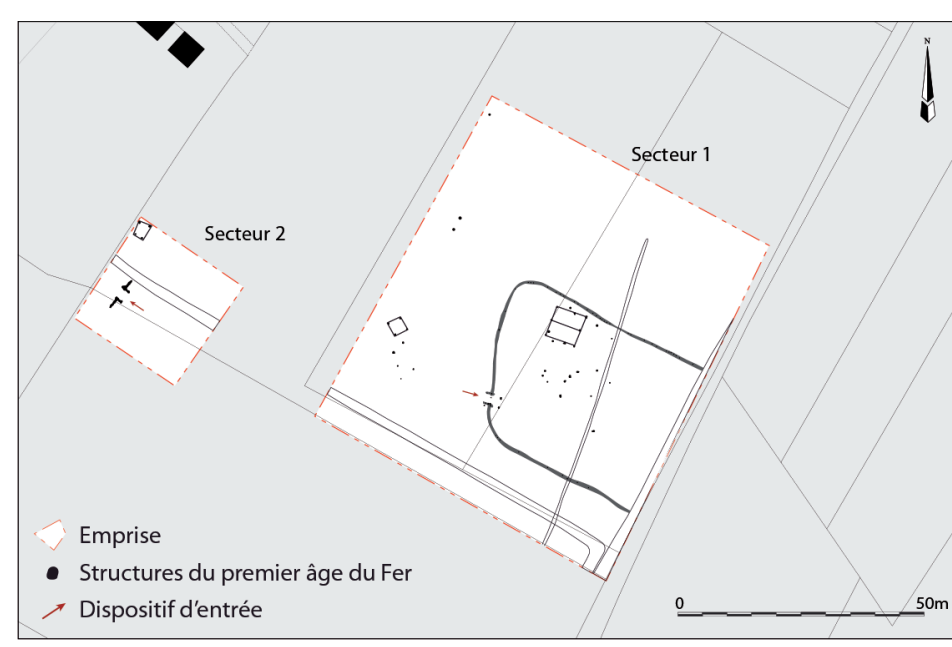

I 4 Les Drouillards à Dompierre-sur-Mer, Charente-Maritime. Plan de l'habitat du premier âge du Fer (d'après Maitay 2012).

quatrième côté n'a pu être mise en évidence au cours de l'évaluation. Le segment oriental a pu ne jamais être aménagé, ou bien être formé de structures peu profondément ancrées dans le sol (talus de terre aujourd'hui arasé, haie ou clôture sur piquets).

\subsection{Les dispositifs d'entrée}

Des systèmes d'entrées définis par deux petites tranchées parallèles dirigées vers l'extérieur de l'enclos constituent l'une des caractéristiques récurrentes des enclos du premier âge du Fer du Centre-Ouest (fig. 8). Aux Drouillards, ces tranchées déterminent un espace de $1,80 \mathrm{~m}$ de largeur, c'està-dire suffisant pour permettre aux hommes et aux bêtes de pénétrer à l'intérieur de l'enclos (fig. 9). Cette interruption peut également être empruntée par des chars ou des charrettes, dont l'écartement des roues à l'âge du Fer varie en moyenne entre 1,10 et $1,30 \mathrm{~m}$ (Buchsenschutz 2009). Les intersections entre ces deux bras parallèles et la tranchée palissadée sont occupées par deux trous de poteau d'un diamètre moyen de $0,30 \mathrm{~m}$, dont l'importance laisse à penser qu'ils étaient reliés par un linteau. Ils peuvent correspondre aux supports d'un portique marquant physiquement l'entrée de la ferme. Ils peuvent également fonctionner, et avec plus de vraisemblance, avec les deux trous de poteau quadrangulaires aménagés dans le prolongement interne des deux bras de l'entrée. Ces quatre éléments verticaux serviraient ainsi de support à un porche d'entrée pouvant être coiffé d'une toiture légère à double pente, comme cela a été restitué pour l'habitat du Pédeau (Lejars et al. 1990, fig. 13). La taille des poteaux ne permet pas de reconstituer un porche monumental censé être vu de loin, voire indiquer le statut du propriétaire, mais correspond davantage à une structure marquant ce qui semble aujourd'hui constituer l'unique entrée de l'enclos. Sa superficie au sol couvre une surface rectangulaire d'environ $15 \mathrm{~m}^{2}$. Un trou de piquet installé entre les deux bras de l'entrée participe sans doute à un système de fermeture ou de support à une barrière pivotante permettant de clore l'espace interne.

Sur le même site, une seconde entrée est déconnectée de toute structure de clôture implantée dans le sol (fig. 4 et 8). Seul un trou de poteau situé dans le prolongement du bras nord complète le dispositif. Aménagée à $80 \mathrm{~m}$ à l'ouest de l'enclos palissadé, l'entrée est orientée en direction du sud-est. Son plan et son profil sont très proches de ceux de l'entrée orientale mais ses dimensions sont sensiblement plus importantes (les deux bras encadrent un espace de $1,85 \mathrm{~m}$ ). Elle a très probablement pu servir, à la manière de l'entrée orientale, d'ancrage à un petit porche sur semelle ou sur poteaux de bois. Cette structure, dont le comblement est daté de la phase moyenne du premier âge du Fer, s'inscrit dans le prolongement de l'entrée est, à laquelle elle peut être reliée par un chemin. La similitude entre les plans et les remplissages de ces deux structures nous invite à proposer un fonctionnement synchrone de ces deux occupations.

Un aménagement présentant une organisation proche et également isolé de toute structure de clôture conservée a été récemment découvert sur le site des Grands Philambins à Chasseneuil-du-Poitou dans la Vienne (Maitay et al. 2012, 47-49, fig. 25-26). Daté de l'âge du Bronze final IIIb, ce système d'entrée est constitué de deux tranchées en forme de $\mathrm{T}$ encadrant un passage de 2,20 $\mathrm{m}$ de largeur (fig. 8). En formant deux barrières latérales, ces structures permettent d'individualiser l'entrée des enclos. Elles permettent de canaliser le troupeau vers l'entrée de l'enclos, voire de servir au décompte du bétail ou à l'isolement de certaines bêtes (Malrain, Blancquaert 2009, 28).

Sur d'autres sites, l'entrée de l'enclos semble marquée par une simple interruption de la palissade (le Coteau de Montigné à Coulon), ou dans le cas des enclos sur file de poteaux, par la présence de deux trous de poteau de tailles plus conséquentes (les Fioux à Villognon : fig. 6).

\subsection{Les partitions internes}

Plusieurs occupations rurales, qu'elles soient ouvertes ou fermées, ont livré des restes de palissades internes dont 


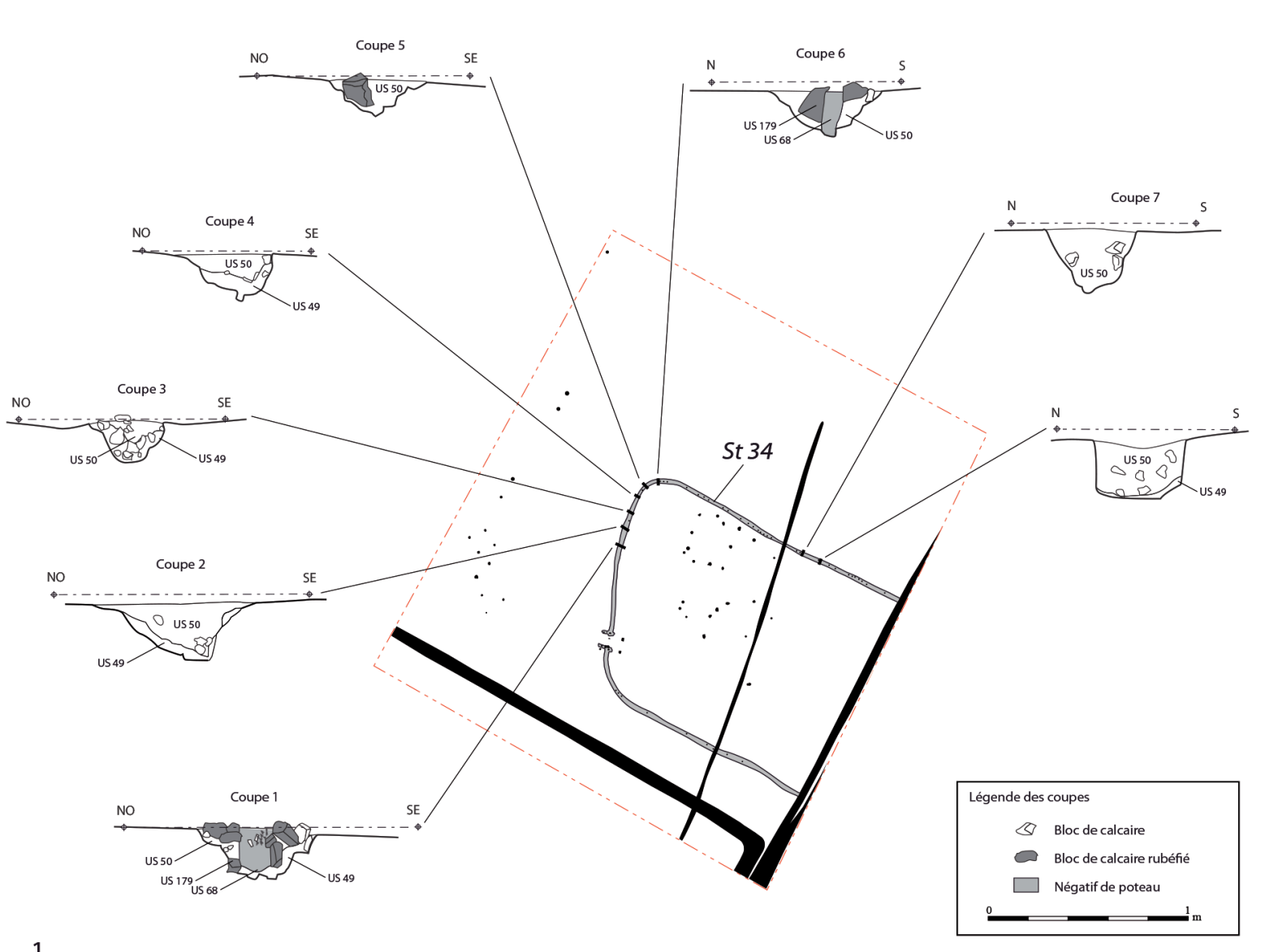

1
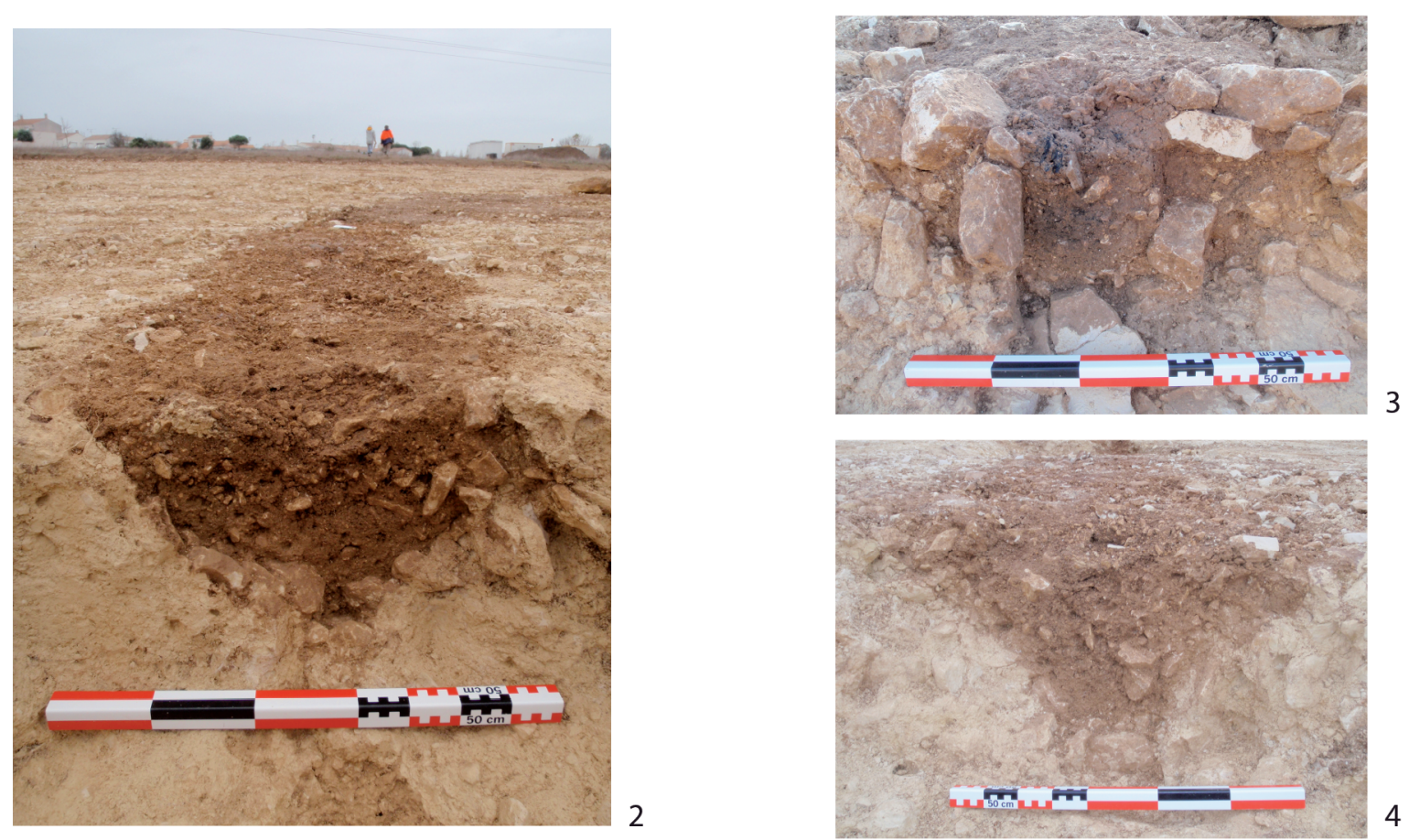

I 5 Enclos palissadé des Drouillards à Dompierre-sur-Mer, Charente-Maritime (clichés Maitay, Inrap). 1 : coupes stratigraphiques de la tranchée de fondation de l'enclos (DAO C. Maitay et V. Sanchez, Inrap) ; $2:$ vue de la tranchée en cours de fouille ; $3:$ vue de l'un des supports verticaux de la palissade (TP 70) ; 4 : vue de la coupe 2. 


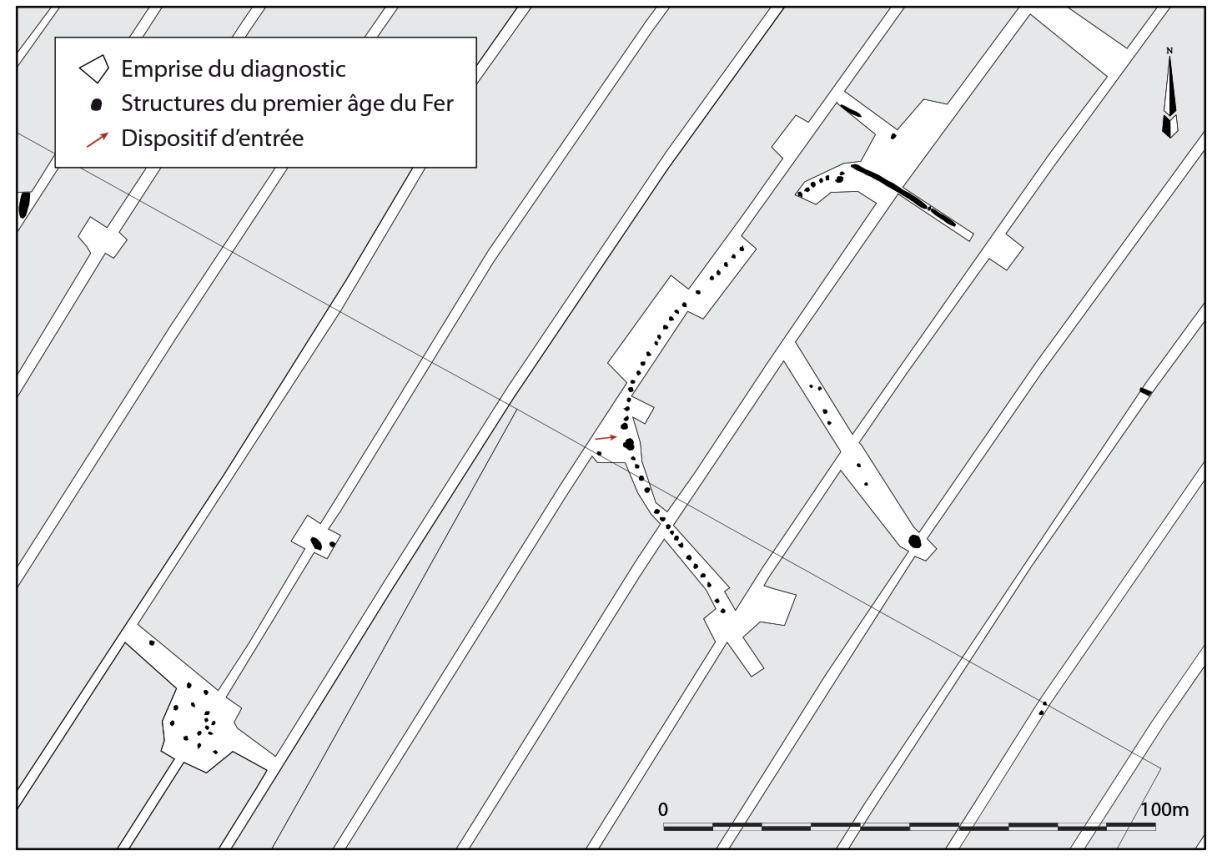

I 6 Le Pédeau à Préguillac, Charente-Maritime. Plan de l'habitat du premier âge du Fer (DAO C. Maitay, d'après Lejars et al. 1990, fig. 11). ne subsistent aujourd'hui que des traces conservées dans le substrat. Comme pour les enclos, deux types principaux de partition interne cohabitent, ceux sur poteaux de bois, les mieux documentés, et ceux sur tranchées de fondation. Des exemples arasés, et certainement incomplets, ont été repérés sur les sites de la Brangerie 1, 2A, 2C et 3 (Pueyo et al. 2013, 161 ; Bardot 2013, 186, 208 et 223) à Villognon, de Malaguet à Migné-Auxances (Sartou, Delalande et al. 2012, 45, pl. II-3-2) ou du Bas des Terres Rouges à Ingrandes (Le Clézio, Giovannacci 2014, fig. 2). On rencontre également des systèmes mixtes alliant alignements de poteaux et tranchées linéaires.

L'habitat des Petits Clairons II nous fournit les exemples de partitions internes les plus pertinents. Une première palissade complexe (ensemble 4) a été fouillée sur une vingtaine de mètres de longueur dans la partie centrale de l'occupation (Baigl et al. 1999, 39, fig. 6) (fig. 3). Elle est consti-

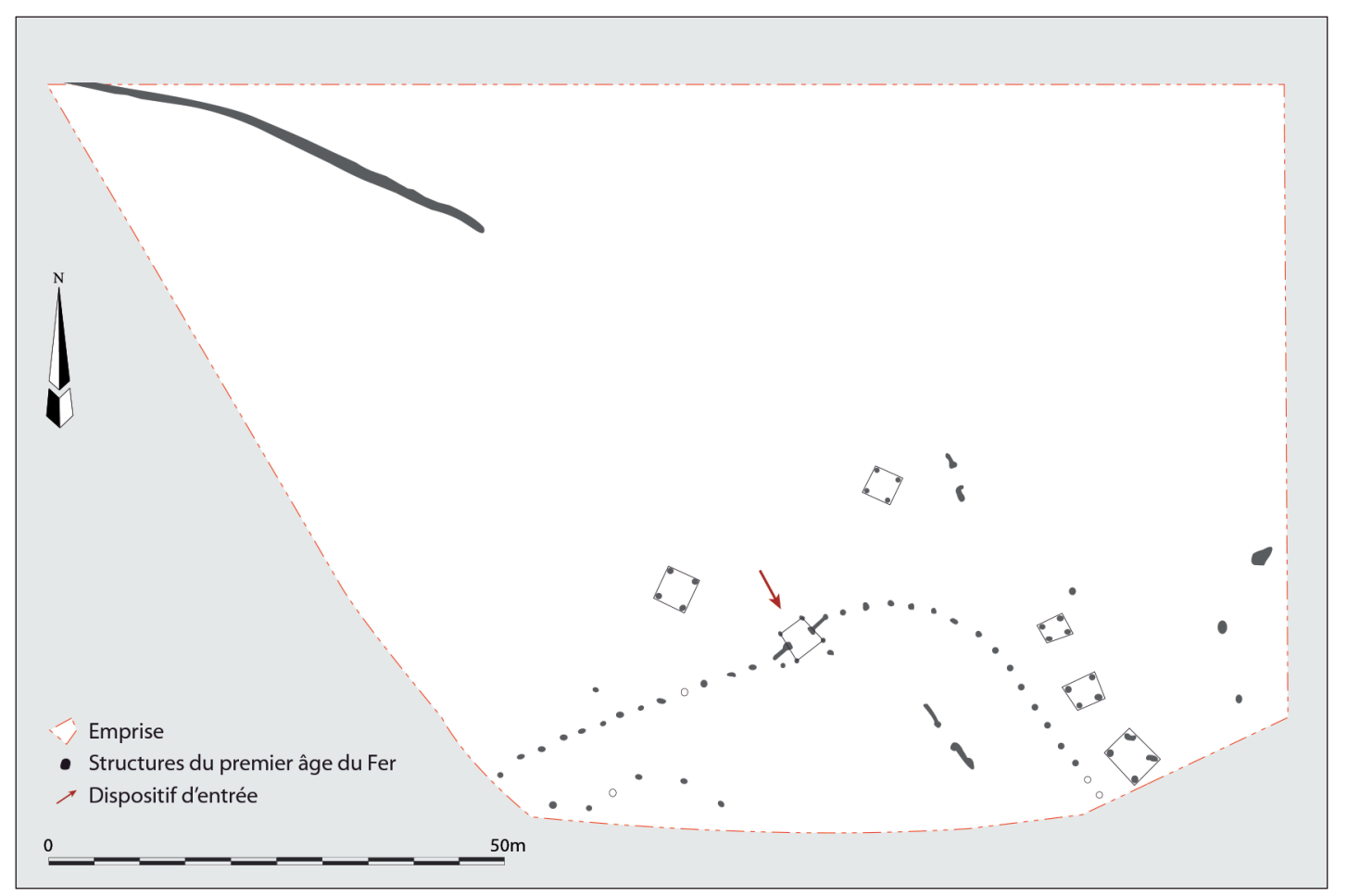

I 7 Les Fioux à Villognon, Charente. Plan des structures de l'âge du Fer observées au diagnostic (DAO C. Maitay, d'après Bats, Pueyo 2011, fig. 13). La flèche rouge indique l'entrée probable de l'enclos. 


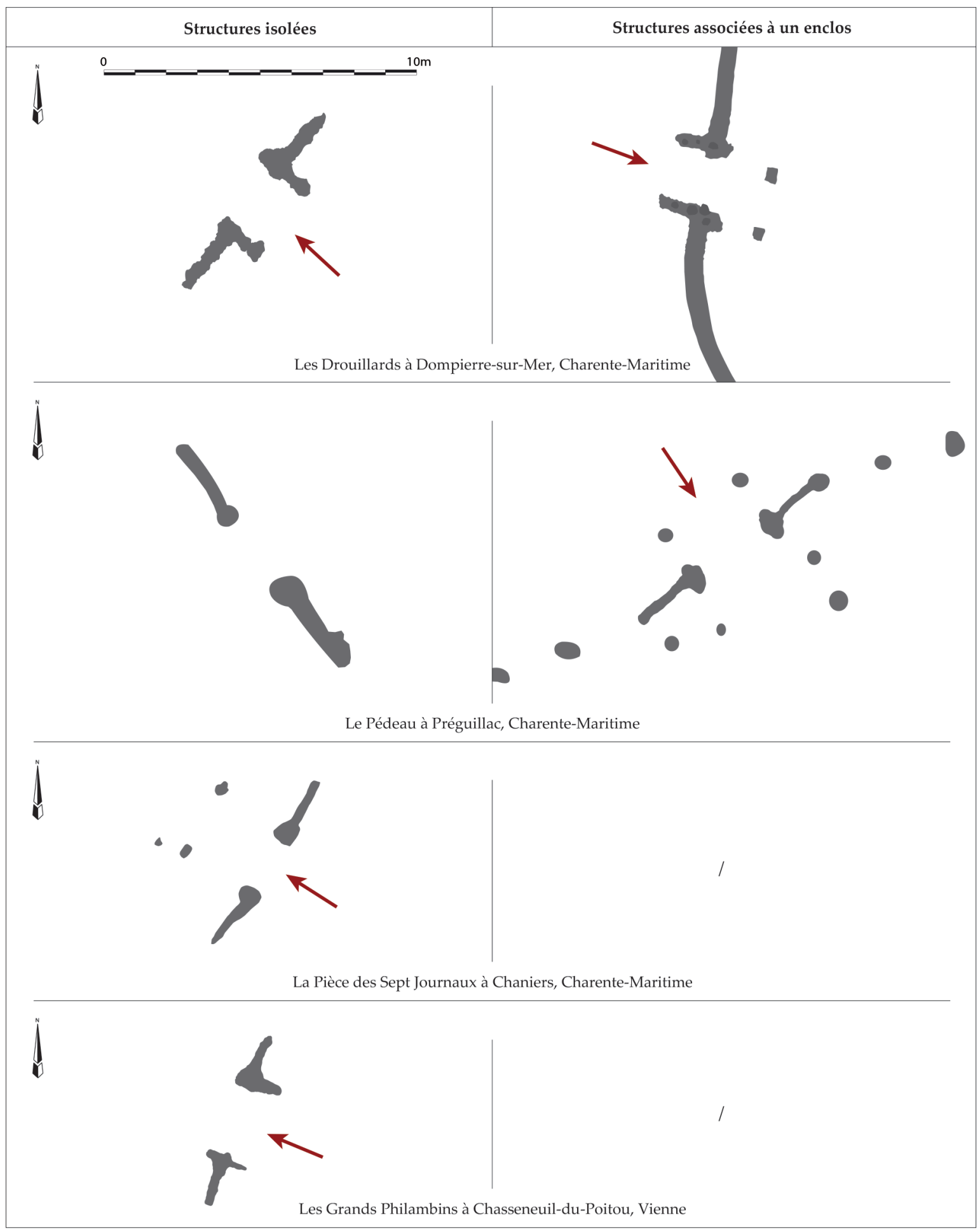

I 8 Systèmes d'entrée observés sur des occupations rurales du premier âge du Fer et de La Tène ancienne (DAO C. Maitay). Deux types principaux se distinguent, celui où l'entrée paraît aujourd'hui déconnectée d'un enclos palissadé (présence d'une haie ou d'une clôture légère) et celui où l'entrée est partie intégrante de l'enclos palissadé.

tuée de neuf poteaux principaux renforcés par quelques supports verticaux secondaires. Dans son prolongement occidental, deux fosses oblongues de $5 \mathrm{~m}$ de long et de 0,20 à $0,30 \mathrm{~m}$ de profondeur conservée définissent un passage de $1,50 \mathrm{~m}$ de lar- geur. L'extrémité de ces structures excavées, de part et d'autre de l'interruption, est renforcée par un creusement circulaire de $0,55 \mathrm{~m}$ de diamètre et une concentration de blocs de calcaire formant un calage. Ce dispositif, qui reçoit deux poteaux de bois 

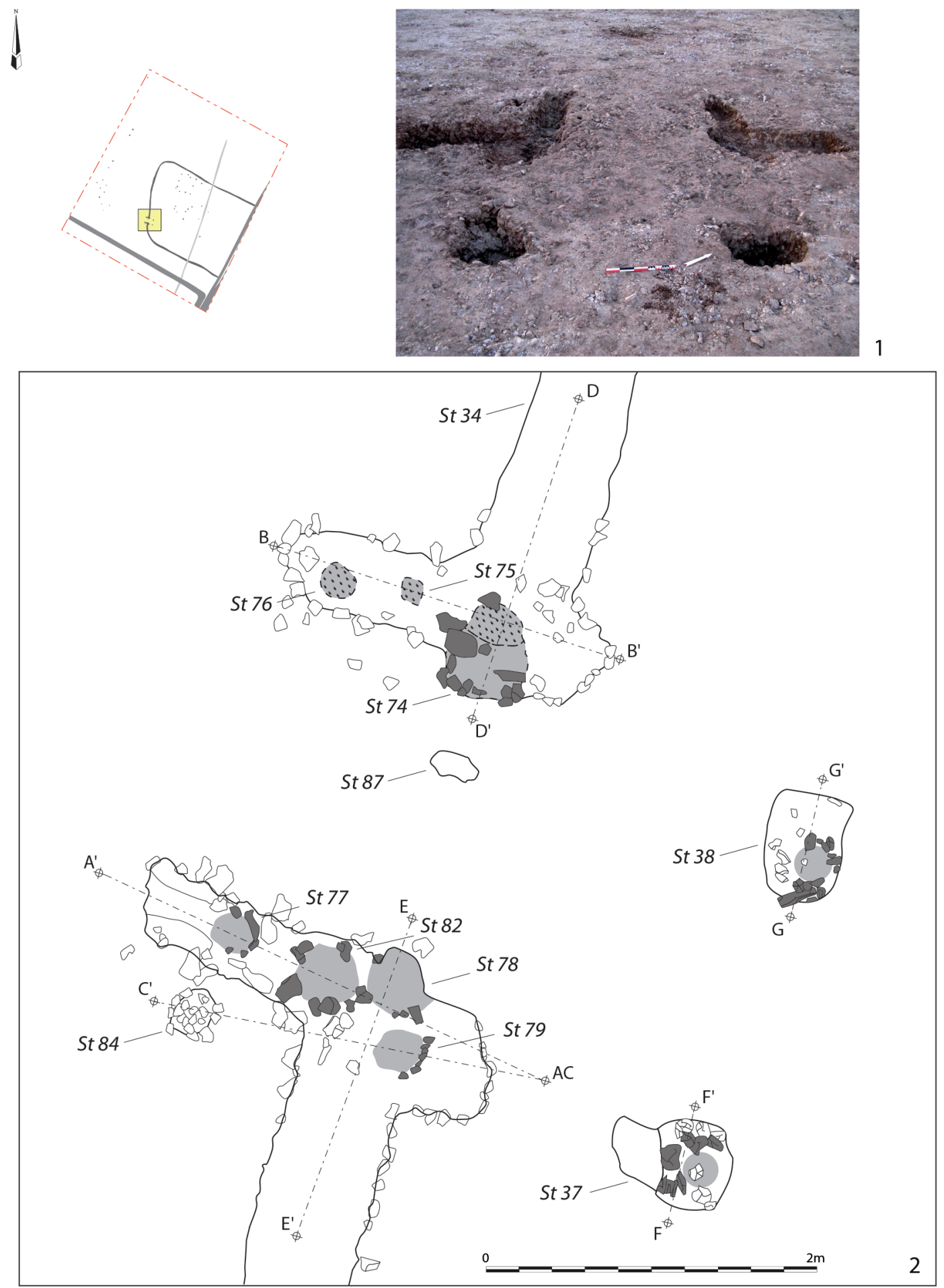

I 9 Les Drouillards à Dompierre-sur-Mer, Charente-Maritime. Vue (1) et plan (2) de l'entrée de l'enclos palissadé (cliché C. Maitay, relevé B. Brisach, DAO V. Sanchez, Inrap).

encadrant l'entrée, constitue certainement un porche ou, plus modestement, un portail. Les deux tranchées se faisant face ont par ailleurs pu recevoir une semelle destinée à accueillir des supports de bois verticaux. Cet ensemble pourrait circonscrire, avec une seconde palissade rectiligne se développant plus au sud, un secteur réservé au stockage des récoltes.
Le second exemple, provenant également des Petits Clairons II (ensemble 11), s'organise par rapport à un alignement de quinze trous de poteau dessinant une structure rectiligne de $35 \mathrm{~m}$ de long orientée est-ouest (ibid., 39-40, fig. 7) (fig. 3). L'extrémité occidentale s'interrompt à environ un mètre de la grande tranchée palissadée. L'entraxe entre chaque 
poteau varie entre 2,50 et $3 \mathrm{~m}$. Deux segments perpendiculaires à l'ensemble 11 se développent au sud de celui-ci. Constitués à la fois d'alignements de trous de poteau et de tranchées de fondation, ils encadrent un espace quadrangulaire d'environ $350 \mathrm{~m}^{2}$ accueillant plusieurs bâtiments sur poteaux porteurs. L'érosion ayant fortement altéré ces structures, la restitution du côté sud repose davantage sur une hypothèse que sur des observations archéologiques. À l'ouest de cet enclos quadrangulaire et au sud de la palissade 11 , les structures excavées sont rares. Cet espace non bâti en marge de l'habitat pourrait très certainement être dévolu à l'agriculture ou au pacage d'animaux domestiques.

\section{L'architecture domestique}

Les bâtiments dont l'attribution chronologique au premier âge du Fer est assurée sont peu nombreux et souvent mal conservés. Une trentaine seulement, dont ne subsistent aujourd'hui que les excavations recevant les poteaux porteurs, a pu être inventoriée. Les trois quarts correspondent à des petits édifices de plan quadrangulaire probablement destinés au stockage des récoltes de grains ou de fourrage (fig. 10). Aucun niveau de sol n'a jusqu'à présent pu être associé à de telles constructions. De récentes opérations de fouille préventive, notamment celles conduites à la Brangerie 2 et 3, en Charente (Bardot 2013), et aux Gains, dans la Vienne (Maguer et al. 2014), favorisent néanmoins l'émergence d'informations sur l'architecture de terre et de bois. On observe dans la région Poitou-Charentes, pour l'âge du Bronze final (Kerouanton et al. sous presse) et le premier âge du Fer, le recours exclusif à des édifices de plan quadrangulaire (fig. 10). Les orientations et les superficies couvertes sont différentes d'un bâtiment à l'autre ${ }^{2}$, et varient de quelques mètres carrés à plus d'une trentaine. On distingue des bâtiments de petit $\left(<12 \mathrm{~m}^{2}\right)$, moyen (entre 12 et $\left.30 \mathrm{~m}^{2}\right)$ et grand module $\left(>30 \mathrm{~m}^{2}\right)$ (Maguer, Robert 2013, fig. 2), dont on peut penser qu'ils remplissent des fonctions différentes.

\subsection{Les bâtiments de grand module : des structures d'habitat?}

Comme plus tard au second âge du Fer (ibid.), les charpentes s'appuient sur des paires de poteaux de bois, généralement au nombre de deux (bâtiments à quatre poteaux) ou de trois (bâtiments à six poteaux). Le modèle circulaire, notamment attesté en Normandie et dans les îles Britanniques, est à ce jour inconnu dans le sud-ouest et le centreouest de la France. Dans ces régions, ces bâtiments n'ont été qu'exceptionnellement découverts accompagnés de tranchées de parois, de niveaux de sols ou de foyers. L'interprétation en tant qu'habitation ne repose donc souvent que sur l'observation de la superficie de l'édifice ou sur la présence à proximité de celui-ci d'un dépotoir. Par ailleurs, l'étroitesse des fenêtres de décapage ne permet que rarement de statuer sur la position privilégiée d'un édifice au sein d'un groupe de constructions (les Petits Clairons II, ensemble 14-15) (fig. 3).

Le bois et la terre constituent les matériaux de construction les plus usités. La pierre n'est visiblement employée, parfois en réemploi (fragments de meules), que pour le calage de supports de bois verticaux. Le chêne semble l'essence la plus fréquente. La découverte de petits fragments d'argile cuite laissant deviner l'empreinte d'éléments de bois confirme l'utilisation de parois en torchis.

Le bâtiment des Drouillards se situe à l'intérieur de la zone délimitée par une palissade, le long du segment nord (Maitay 2012, fig. 5) (fig. 10, $\mathrm{n}^{\circ}$ 2). Le plan se compose d'une seule nef orientée sud-est/nord-ouest et constituée de deux travées oblongues. Huit trous de poteau, répartis en deux rangées de trois et une rangée médiane de deux, définissent un espace d'environ $35 \mathrm{~m}^{2}$. Ils adoptent une forme généralement circulaire aux parois verticales ou évasées ; certains possèdent un plan quadrangulaire aux angles arrondis. Les comblements, très homogènes d'une structure à l'autre, sont constitués par des sédiments argilo-limoneux meubles contenant quelques graviers et cailloux émoussés. Plusieurs trous de poteau ont conservé la marque en négatif de poteaux de bois ainsi que leurs systèmes de calage. L'emplacement des poteaux est matérialisé par un espace circulaire vide de blocs de pierre et de cailloux, et colmaté par un sédiment de couleur brun gris aéré et organique (présence de petits charbons de bois). Ils peuvent être centrés dans la structure ou bien légèrement décalés. Leurs diamètres varient et semblent correspondre à deux principaux modules (l'un d'environ 0,20 à $0,25 \mathrm{~m}$ de diamètre, l'autre supérieur à $0,35 \mathrm{~m}$ de diamètre).

L'examen du plan des Petits Clairons II et de la documentation de fouille permet de proposer la restitution d'un nouveau bâtiment à poteaux porteurs (fig. 10, no 4 ). Les ensembles 14 et 15 , au sud de la palissade 11, ont été présentés initialement comme deux greniers distincts. Nous proposons aujourd'hui un grand édifice à une nef et quatre travées, d'orientation nord-sud. La construction, qui pour- 


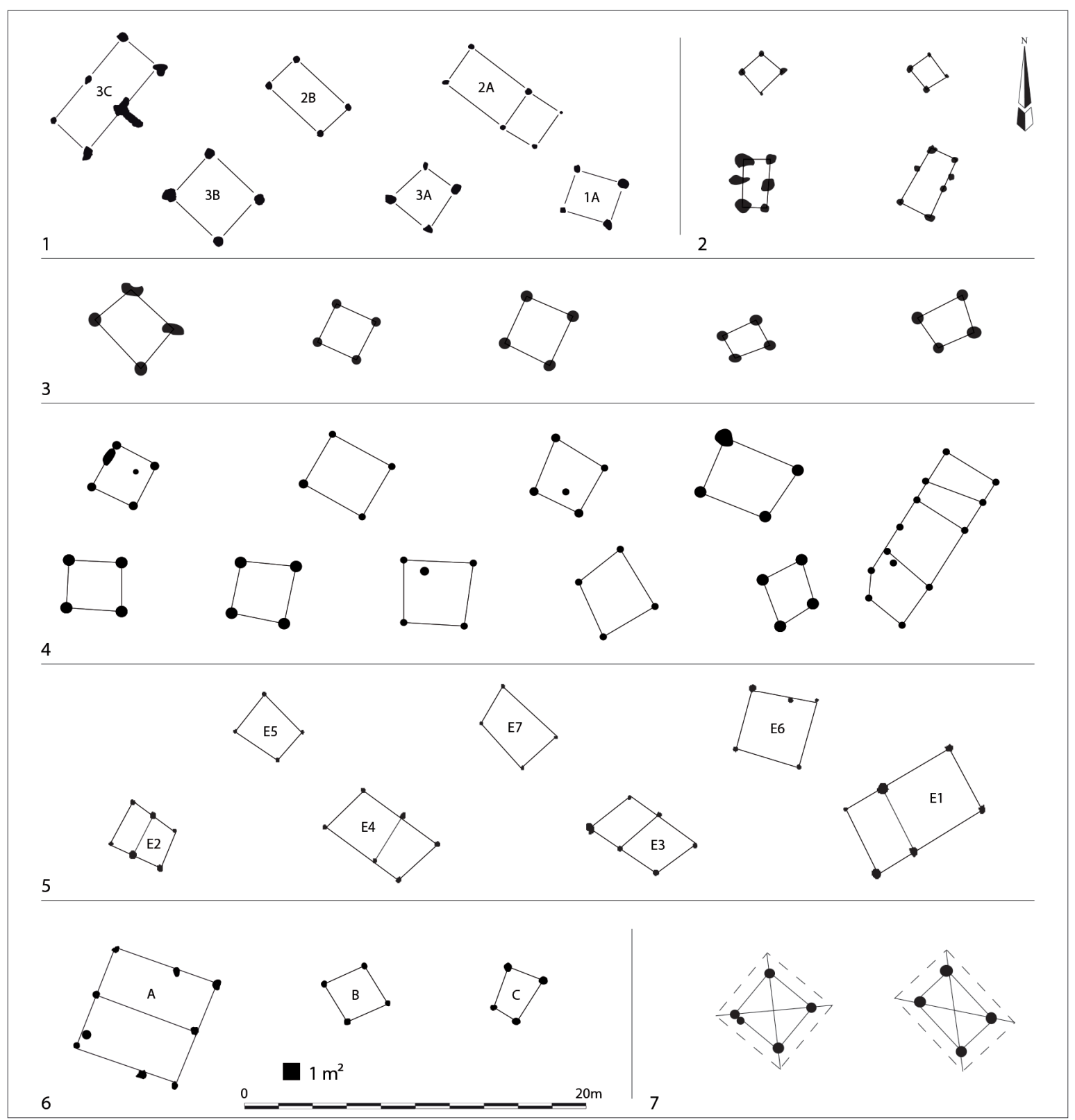

10 Plans de bâtiments sur poteaux du premier âge du Fer (DAO C. Maitay). 1 : Le Coteau de Montigné à Coulon, Deux-Sèvres, transition Bronze final III-premier âge du Fer (d'après Pautreau 1981 et 1983) ; 2 : Les Drouillards à Dompierre-sur-Mer, Charente-Maritime, phase moyenne du premier âge du Fer (d’après Maitay 2012) ; 3 : Le Pédeau à Préguillac, Charente-Maritime, phase moyenne du premier âge du Fer (d'après Lejars et al. 1990, fig. 11) ; 4 : Les Petits Clairons II à Barbezieux, Charente, phase moyenne du premier âge du Fer (d'après Baigl et al. 1999, fig. 4) ; 5 : Le Mas de Champ Redon à Luxé, Charente, phase moyenne du premier âge du Fer (d'après Audé dir. 2013) ; 6 : La Fontaine 1 à Reignac-Barbezieux, Charente, phase finale du premier âge du Fer-La Tène ancienne (d'après Landreau et al. 2009, fig. 4).

rait disposer d'un angle coupé au sud-ouest, reposerait alors sur cinq paires de poteaux et couvrirait une superficie d'environ $32 \mathrm{~m}^{2}$ (3,30 x 9,60 m). Des trous de poteau isolés ont été découverts dans l'environnement de ce bâtiment mais aucune trace de foyer ou de niveau d'occupation n’a été observée.

La fouille de l'habitat du second âge du Fer des Gains à Saint-Georges-Lès-Baillargeaux dans la Vienne (Ma- guer et al. 2014) a été l'occasion de mettre au jour les fondations de deux bâtiments datés de la phase finale du premier âge du Fer (E23 et E24). De dimensions (4,40 x $11,25 \mathrm{~m}$, soit une superficie de $49,5 \mathrm{~m}^{2}$ ) et d'orientation (nord-ouest/sud-est) identiques, ils possèdent tous les deux un plan quadrangulaire formé par trois files de quatre poteaux. Il s'agit pour l'instant des seuls exemples d'architectures à deux nefs du premier âge du Fer connus dans le Centre-Ouest. 
Enfin, la fouille de l'habitat de La Tène ancienne de la Fontaine 1 à Reignac-Barbezieux en Charente (Landreau et al. 2009, 250-252, fig. 4) a livré les plans de deux édifices sur poteaux de $12 \mathrm{~m}^{2}$. Chacun a pu être utilisé, si l'on retient l'hypothèse de bâtiments à parois rejetées hors du module porteur, en tant qu'habitation (fig. 10, $n^{\circ} 6$ ).

\subsection{Les bâtiments de moyen module: des constructions à vocation agricole?}

Des bâtiments de taille inférieure (entre 12 et $30 \mathrm{~m}^{2}$ ) et dont l'ancrage au sol est généralement plus modeste (trous de poteau de diamètre et de profondeur moins élevés) sont généralement interprétés, faute de mieux, comme des bâtiments annexes. Il peut s'agir de resserres, de granges ou de préaux, ou de constructions destinées à accueillir des animaux domestiques (bergeries, écuries).

Deux bâtiments rectangulaires du Bronze final IIIb, couvrant environ $17 \mathrm{~m}^{2}$ chacun, ont pu être fouillés dans les secteurs 2 et 3 des Grands Philambins à Chasseneuil-du-Poitou dans la Vienne (Maitay et al. 2012, fig. 71 et 72). Le bâtiment 2A, d'orientation sud-est/nord-ouest, est constitué d'une travée oblongue se poursuivant par une seconde plus petite de plan carré. Cette dernière, marquée par des trous de poteau de taille modeste, pourrait être le support d'un appentis s'appuyant sur l'un des murs pignon du bâtiment. Le bâtiment $3 \mathrm{C}$, d'orientation sud-ouest/nord-est, rassemble quant à lui deux modules de taille identique $\left(8,5 \mathrm{~m}^{2}\right)$. Les parois longues mesurent dans les deux cas 6,40 $\mathrm{m}$ de longueur, et les pignons 2,65 (2A) et 2,72 m (3C). Les plans de ces constructions, tels qu'ils nous sont parvenus, pourraient correspondre à ceux de petites structures d'habitat. En revanche, si l'hypothèse de constructions à vocation domestique est privilégiée (petites maisons, resserres, annexes...), celle de greniers, non pas sur quatre mais sur six poteaux, doit également être considérée.

Quelle que soit la fonction retenue, quelques rares constructions de plan et de superficie similaires ont pu être recensées sur des occupations du premier âge du Fer, comme aux Ormeaux à Angoulins en Charente-Maritime (Maguer et al. 2009, 60, fig. 4). Quatre des sept bâtiments quadrangulaires de Champ Redon (fig. 10, $\mathrm{n}^{\circ}$ 5) ont une superficie comprise entre 14 et $18 \mathrm{~m}^{2}$ (E3, E4, E6 et E7) : ils sont interprétés comme des bâtiments agricoles de type remise (Audé et al. 2013a, 107-112). Le bâtiment à six poteaux E7, d'une superficie de $28 \mathrm{~m}^{2}(7 \times 4 \mathrm{~m})$, peut quant à lui être considéré comme un probable bâtiment d'habitation. Ses fondations se distinguent nettement de celles des édifices précédents (trous de poteau plus larges et plus profonds, calages de pierres organisés en couronne). D'autres édifices, datés cette foisci de La Tène A2-B1, pourraient également être liés à des activités agricoles de stockage ou de parcage (la Brangerie à Villognon : Bardot 2013). Mais, une fois encore, l'absence de niveau d'occupation conservé invite à considérer l'attribution fonctionnelle de ces bâtiments avec une grande prudence...

\section{Le stockage des denrées périssables}

Les informations disponibles sur les modalités de stockage dans le centre-ouest de la France (jarres en terre cuite, greniers et silos) restent depuis une enquête menée à la fin des années 1990 (Pautreau, Gomez de Soto 1999) encore indigentes. Les structures observées sur le terrain sont peu nombreuses et ne livrent qu'exceptionnellement des restes végétaux permettant de discuter des produits stockés et de leur consommation. Dans l'état actuel des données, le stockage des récoltes s'effectue préférentiellement hors sol, dans des greniers surélevés.

\subsection{Les jarres en terre cuite}

Au premier âge du Fer, les formes des vases en terre cuite destinés au stockage sont héritées de celles de l'âge du Bronze final (fig. 11) (Maitay et al. 2009a). De grands vases à panse piriforme ou ovoïde, fond plat et haut col cylindrique ou évasé se retrouvent fréquemment sur les habitats de la région, notamment aux Petits Clairons II (Baigl et al. 1999, fig. $20,25, \mathrm{n}^{\mathrm{os}} 89,28, \mathrm{n}^{\mathrm{0}} 188$, etc.). Le bord peut être replié vers l'extérieur (pour faciliter le maintien d'un couvercle ?) et l'encolure renforcée d'un ou de plusieurs cordons digités ou incisés. Certains vases à panse tronconique, épaulement haut placé et col évasé, de capacité moindre, ont également pu être utilisés (ibid., fig. 19, $\mathrm{n}^{\text {os }} 29,26, \mathrm{n}^{\circ} 167$ ) (fig. 11, $\mathrm{n}^{\circ}$ 6). Ces récipients proviennent principalement du comblement de fosses abandonnées mais aussi de petits creusements dans lesquels ils étaient disposés (vases « silos »).

L'originalité de ces productions se manifeste dans la proportion de vases décorés, relativement importante au regard de l'ornementation des autres types de poteries du premier âge du Fer (Maitay et al. 2009a). Le vocabulaire stylistique repose principalement sur les digitations, disposées sur un cordon plastique horizontal faisant par ailleurs office de renfort, ou imprimées directement sur la paroi du vase, 

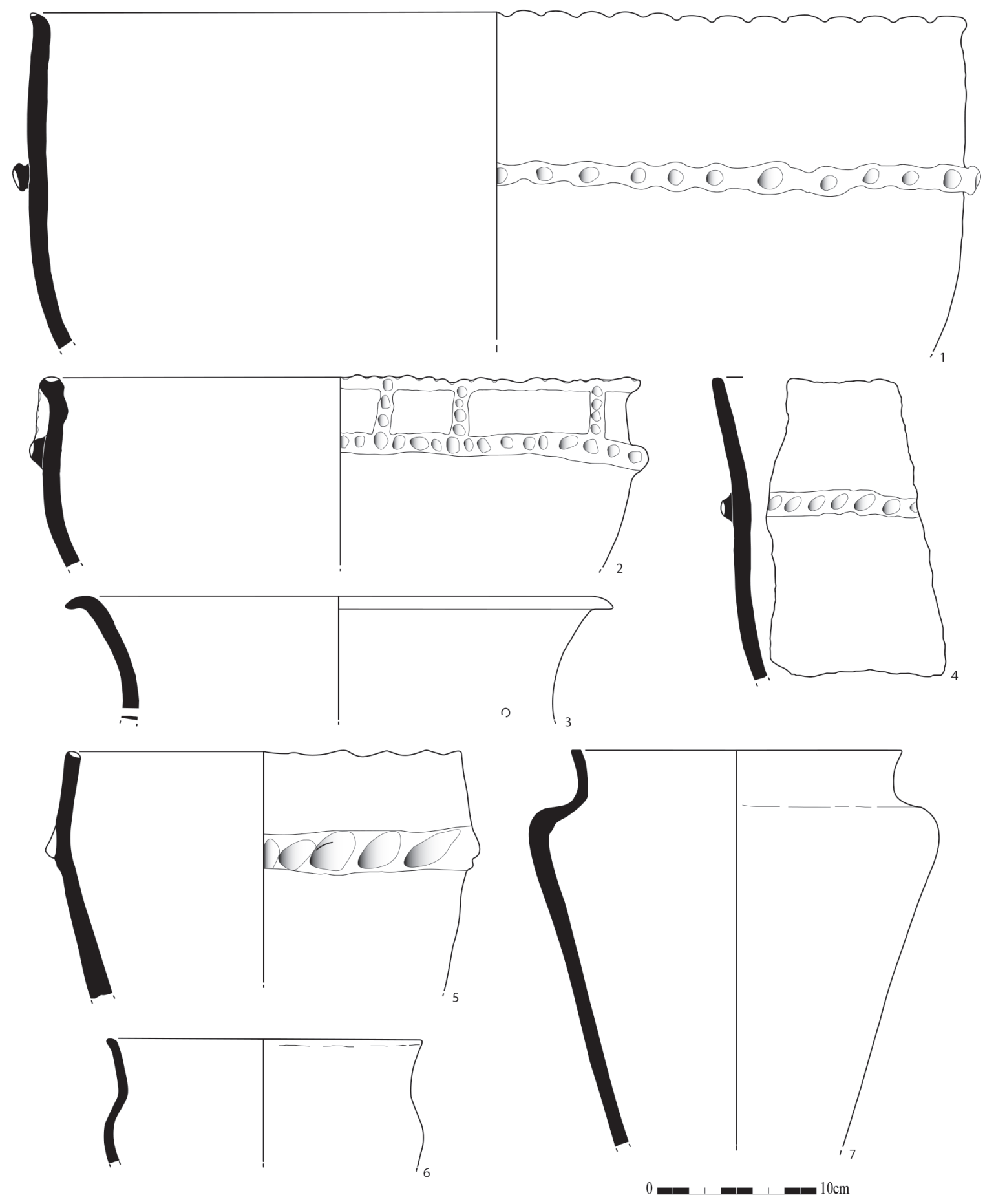

11 Vases de stockage. 
également en registres horizontaux (fig. $11, \mathrm{n}^{\text {os }} 1,2,4$ et 5). L'impression digitée agrémente ainsi fréquemment la lèvre (fig. $11, \mathrm{n}^{\text {os }} 1,2$ et 5 ) et la jonction col-panse des récipients à encolure. On observe par ailleurs, comme au Mas de Champ Redon (Audé et al. 2013a, fig. 125) ou aux Grands Champs à Coulon, dans les Deux-Sèvres (fig. 11, $\mathrm{n}^{\circ} 2$ ), quelques cas de cordons digités répartis sur le col en registres horizontaux et verticaux.

\subsection{Les silos}

Les structures de stockage excavées abondamment utilisées par les populations de l'âge du Bronze ancien (la Viaube à Jaunay-Clan, dans la Vienne : Lavoix et al. 2013 ; Maitay en préparation) puis final (les Varennes à Saint-Georges-LèsBaillargeaux : Fouéré et al. 2011 ; la Viaube, dans la Vienne : Maitay en préparation) ne se retrouvent qu'exceptionnellement sur les occupations rurales du premier âge du Fer du Centre-Ouest. De nombreuses fosses aux formes variées sont connues dans la région mais seulement quelques rares exemplaires interprétés comme des silos peuvent être retenus. Un premier exemple, découvert en 1998 lors de la fouille de la nécropole néolithique de la Jardelle à Dissay dans la Vienne, possède une ouverture sub-quadrangulaire d'1,20 m de diamètre, un profil tronconique et une profondeur conservée d'1,10 m (fig. $\left.12, n^{\circ} 1\right)$. Son originalité réside dans la pré- sence d'une structure de couverture portée par quatre paires de poteaux de bois (Pautreau, Gomez de Soto 1999, 333-334, fig. 1). Le mobilier céramique présent dans les niveaux de remplissage atteste d'un comblement intervenant au début de La Tène ancienne. Ce système de superstructure en bois protégeant l'ouverture d'un silo a également été observé sur le site d'habitat du début du second âge du Fer de Terre-quiFume à Buxerolles dans la Vienne (Maguer, Pautreau 2007, fig. 1). Un autre silo moins bien conservé a été fouillé sur le tracé de la future ligne LGV SEA (Audé et al. 2013a, 127, fig. 106). Son ouverture, nettement plus évasée que pour celui de la Jardelle, s'explique par un effondrement progressif des parois, avant une ultime phase de comblement volontaire (fig. 12, $\mathrm{n}^{\circ}$ 2). Un dernier exemple vient d'être récemment mis en évidence lors d'une opération de diagnostic réalisée dans le nord des Deux-Sèvres. Ce silo dispose d'une ouverture ovalaire de $1,56 \mathrm{~m}$ de longueur pour 1,20 $\mathrm{m}$ de largeur et d'une profondeur conservée de $0,7 \mathrm{~m}$; son profil est tronconique (Audé et al. 2013b, 36, fig. 31). Il contenait des restes de faune ainsi que des fragments de vases en terre cuite du Hallstatt D1-D2.

\subsection{Les greniers}

Les vestiges archéologiques attestant du stockage aérien des denrées céréalières sont en revanche beaucoup plus

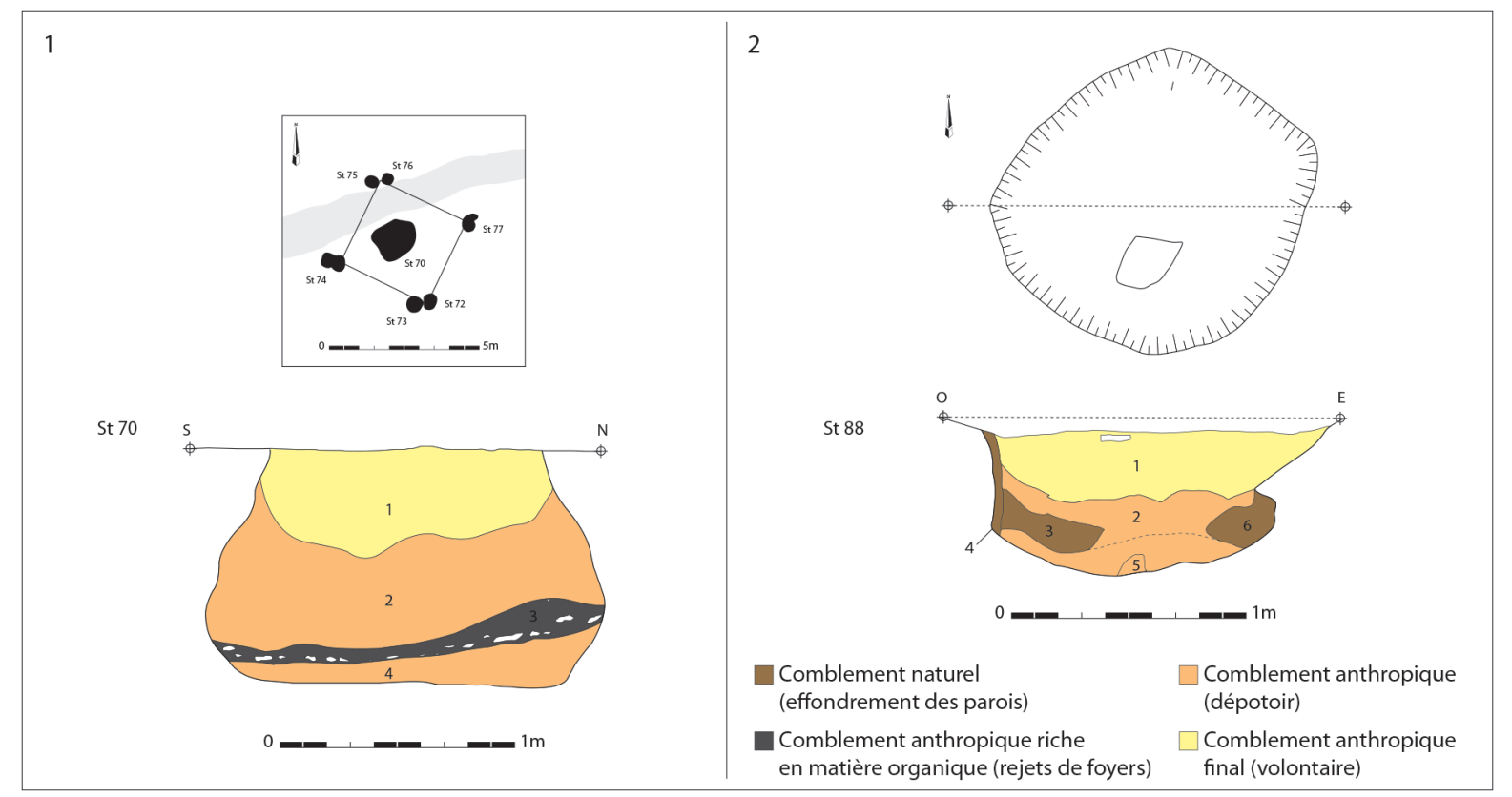

12 Plans et coupes stratigraphiques de silos du premier âge du Fer et de La Tène ancienne (DAO C. Maitay). 1 : La Jardelle à Dissay, Vienne (d'après Pautreau, Gomez de Soto, 1999, fig. 1) ; 2 : Le Mas de Champ Redon à Luxé, Charente (d'après Audé dir. 2013, fig. 106). 
nombreux en Poitou-Charentes. Ils se rencontrent fréquemment sur les sites du Bronze final et leur utilisation privilégiée se poursuit jusqu'à La Tène finale (Pautreau, Gomez de Soto 1999 ; Maguer, Lusson 2009, 432-433). Les exemplaires du premier âge du Fer correspondent aux plans et aux modules de ceux fouillés dans les autres régions de la France (Audouze, Buchsenschutz 1989, 161). Les plans quadrangulaires à quatre poteaux ancrés dans le substrat sont les mieux documentés (le Coteau de Montigné : fig. 10, $\mathrm{n}^{\circ} 1$ ; le Pédeau : fig. $10, \mathrm{n}^{\circ} 3$; la Brangerie ; etc.), mais il en existe également quelques exemples à six ou sept poteaux (le Coteau de Montigné : fig. $10, \mathrm{n}^{\mathrm{o}} 1$ ), voire à neuf (Malaguet : Sartou, Delalande et al. 2012, 48). Les poteaux porteurs disposent d'un diamètre moyen de 0,20 à $0,30 \mathrm{~m}$. Ces greniers peuvent aussi bien se situer à l'intérieur de l'enclos (le Coteau de Montigné) qu'à l'extérieur de la palissade (les Drouillards : fig. 4). Aux Petits Clairons II, ils sont localisés à la fois à l'intérieur d'espaces clos par des alignements de poteaux, et au-delà de la grande palissade sur tranchée, dans des espaces apparemment non construits (fig. 3). Au Pédeau, en Saintonge, quatre greniers quadrangulaires à quatre poteaux sont disposés à une dizaine de mètres à l'extérieur de l'enclos palissadé (fig. 7). Ils occupent des superficies généralement comprises entre 4 et $12 \mathrm{~m}^{2}$.

\section{Les éléments structurants du paysage}

\subsection{Parcellaires et chemins}

Dans le Centre-Ouest, l'installation d'un réseau parcellaire ancien en relation avec les habitats du premier âge du Fer reste inconnue. Aucun exemple de structures linéaires fossoyées définissant des parcelles n'a actuellement pu être mis en évidence, y compris sur les opérations de fouille portant sur de grandes superficies (les Petits Clairons II, les Gains ou Malaguet). Un tel constat, également valable pour l'âge du Bronze et La Tène ancienne, peut s'expliquer, du moins en partie, par des phénomènes d'arasement liés aux pratiques agraires modernes, ou par l'existence de structures non pas en creux mais en élévation (palissades légères, haies vives, talus plantés d'arbres et d'arbustes). Cette dernière hypothèse, renforcée, comme nous l'avons vu plus haut, par la découverte de systèmes d'entrée apparemment isolés semble aujourd'hui envisageable.

Une seule structure linéaire fossoyée, suivie sur une longueur d'environ $50 \mathrm{~m}$, a été observée lors de la fouille du
Pédeau (fig. 7). Elle mesure 0,60 à $0,80 \mathrm{~m}$ de largeur et $0,30 \mathrm{~m}$ de profondeur. Son fond est plat et ses parois évasées. Si la contemporanéité de ce fossé avec l'occupation qui la jouxte ne fait guère de doute, son interprétation en tant qu'élément parcellaire reste à démontrer. L'extrémité orientale qui s'interrompt brutalement marque une légère inflexion en direction de l'entrée de la ferme. Ainsi l'éventualité d'un fossé bordant un chemin permettant l'accès au site est à considérer.

Les vestiges d'un chemin creux associé à quelques fosses et bâtiments sur poteaux de la phase finale du premier âge du Fer ou du début de La Tène ancienne ont été mis au jour sur l'habitat de la ZAC des Ormeaux à Angoulins, en Charente-Maritime (Maguer et al. 2009, 61-62, fig. 3 et 5). D'orientation nord/sud, il est large d'environ $5 \mathrm{~m}$ et possède un profil largement ouvert. Son comblement est constitué d'un niveau de circulation formé de galets, de cailloux et de blocs de calcaire de petit module. Cette structure, dont la mise en place pourrait dater de la fin du premier âge du Fer, est recouverte d'un niveau de fonctionnement constitué de limons très organiques, riches en tessons de poterie (récipients culinaires, godets à sel), restes de faune et malacofaune. Ce chemin est actuellement sans équivalent dans la région.

En effet, la fouille de portions de voies antiques (Malaguet : Sartou, Delalande et al. 2012, 60-65) atteste, pour le moment, de la perduration de voies qui, étonnamment, ne semblent pas antérieures à l'époque gauloise. On peut s'étonner par exemple de la position de l'habitat du Pédeau à quelques mètres seulement de la voie antique reliant Saintes à Bordeaux et qui emprunte certainement le tracé d'une voie plus ancienne.

L'existence d'axes de circulation peut être suggérée à partir de l'observation de la répartition des bâtiments et des palissades au sein de l'habitat. C'est notamment le cas sur l'occupation du Bronze final III de la Viaube 1 (Lavoix et al. 2013, 133-136), ou sur celle, plus récente, des Petits Clairons II (fig. 3).

\subsection{Relations habitats - nécropoles}

Si le cadre de vie des populations rurales du premier âge du Fer semble pouvoir s'esquisser peu à peu, il n'en va pas de même pour les espaces réservés aux défunts. Les nécropoles à enclos fossoyés du premier âge du Fer, abondantes et bien documentées dans la région (Baranger 2009), semblent pour l'instant à l'écart des zones d'habitat, 
qu'il s'agisse de sites de hauteur fortifiés (Maitay et al. 2009b, 396-397) ou d'occupations rurales de plateaux ou de fonds de vallée (Maitay, Nillesse 2014).

Si l'on estime que les terres environnant l'habitat sont réservées à l'exploitation agricole, les nécropoles pourraient quant à elles être reléguées à quelques centaines de mètres des habitations, à la frontière des sols cultivés. L'habitat du Pédeau par exemple comprend dans un rayon de $2500 \mathrm{~m}$ une dizaine de sites à enclos fossoyés repérés par prospection aérienne (Lejars et al. 1990, 8). La nécropole à enclos circulaires et quadrangulaires du Terrier Blanc, à $200 \mathrm{~m}$ au nord-ouest du Pédeau, devait donc également se situer sur le terroir directement exploité par les habitants de la ferme.

\section{Conclusion}

On observe depuis quelques années, grâce aux grands décapages et aux synthèses proposées dans d'autres régions de France, notamment en Normandie (Marcigny 2012) et dans le Languedoc (Séjalon et al. 2009), un regain d'intérêt pour l'occupation des campagnes et les populations rurales protohistoriques. Cette émulation récente touche les campagnes de l'âge du Bronze (Brun, Marcigny 2012) et désormais celles du premier âge du Fer (Desbrosses, Riquier 2012). Ainsi, un modèle théorique de l'établissement agricole du premier âge du Fer regroupant un ou plusieurs bâtiments d'habitation sur poteaux plantés, des structures de stockage (silos ou greniers), des fosses excavées aux fonctions parfois imprécises et des zones non construites interprétées comme des espaces ouverts dévolus à l'agriculture ou à l'élevage peut être défini. L'habitat est généralement clôturé, non défensif et dispersé.

Cette organisation, dont les lignes directrices sont en place dès l'âge du Bronze, connaîtra à partir du III' s. av. J.-C. de profondes modifications avec l'installation en Gaule d'un vaste réseau d'établissements ruraux précédant la mise en place des grands domaines de la fin de La Tène.

Néanmoins si, pour le Centre-Ouest de la France, des tendances générales semblent pouvoir s'esquisser, les modalités d'exploitation des campagnes, les produits cultivés, et par conséquent l'impact du facteur anthropique sur l'environnement, restent encore difficiles à saisir (Maitay, Nillesse 2014). L'extrême indigence des données paléoenvironnementales (analyses anthracologiques, carpologiques et palynologiques), limite aujourd'hui les possibilités d'interprétation des espaces cultivés et des paysages environnants (paysages de bocage, d'openfield ?). Par ailleurs, l'état de conservation du matériel osseux ne permet qu'exceptionnellement d'aborder la place de l'élevage ou les pratiques alimentaires de ces populations.

On s'étonnera également de l'absence de découvertes relatives à la mise en place d'un parcellaire ancien, attesté en Languedoc à la fin du VI ${ }^{\mathrm{e}}$ s. av. J.-C. (Séjalon et al. 2009, 166) et en Normandie dès l'âge du Bronze ancien et le début du Bronze moyen (Marcigny, Ghesquière 2003 ; Marcigny et al. 2007). Plusieurs portions isolées de réseaux fossoyés ont pu être mises au jour dans le cadre de diagnostics ou de fouilles, mais, comme c'est généralement le cas dans d'autres régions de l'Ouest, aucune n'a pu être associée avec certitude à l'âge du Bronze final ni au premier âge du Fer. L'hypothèse d'enclos ou de parcelles déterminés par des haies vives ou des petits talus, plutôt que par des structures fossoyées, devra donc à l'avenir être discutée à partir de nouvelles données de terrain.

Christophe MAITAY Chargé d'opération et de recherche, INRAP GSO, Centre archéologique de Poitiers 
1. Sur les plateaux jurassiques, la faible épaisseur des terres combinée aux méthodes aratoires modernes ne favorisent pas toujours la bonne conservation des sites ; c'est par exemple le cas du plateau aunisien autour de La Rochelle.
2. Les superficies évoquées correspondent à la surface comprise entre les poteaux porteurs, aucun exemple d'édifice à paroi rejetée n'étant attesté dans la région au premier âge du Fer.

Références bibliographiques

Audé et al. 2013a : AUDÉ (V.) dir., avec la coll. de DUPONT (C.), FERRIÉ (J.-G.), LOUYOT (D.), MOUGNE (C.), POIRIER (P.), PAILLÉ (Y.), QUERRÉ (G.), SEMELIER (P.) - Le Mas de Champ Redon à Luxé (Charente), un ensemble sépulcral du Bronze ancien et un habitat du premier âge du Fer, Rapport final d'opération de fouille, Inrap GSO-Inrap LGV SEA, L'Isle-d'Espagnac, 2013, 270 p., 141 fig.

Audé et al. 2013b : AUDÉ (V.) dir., avec la coll. de BAKKAL-LAGARDE (M.-C.), MAITAY (C.) - Un habitat rural du premier âge du Fer. ZEI Bois Roux, SaintAubin-du-Plain, Deux-Sèvres, Rapport final d'opération de diagnostic, Inrap GSO, Poitiers, 2013, 64 p., 49 fig.

Audouze, Buchsenschutz 1989 : AUDOUZE (F.), BUCHSENSCHUTZ (O.) - Villes, villages et campagnes de l'Europe celtique. Paris, Hachette, Bibliothèque d'archéologie, 1989.

Baigl et al. 1999 : BAIGL (J.-P.), avec la coll. de GOMEZ DE SOTO (J.), POIRIER (P.), KEROUANTON (I.), BAYEN (É.) - Barbezieux, Les Petits Clairons (Charente). Un établissement rural du premier âge du Fer, Aquitania, 16, 1999, p. 31-91.

Baranger 2009 : BARANGER (A.) - Enclos et structures funéraires de l'âge du Bronze et de l'âge du Fer en Poitou-Charentes. Mémoire de master II « Civilisation antique et médiévale », Université de Poitiers, UFR Sciences Humaines et Arts, 2009, 2 vol.

Bardot 2013 : BARDOT (X.) - Deux occupations de La Tène ancienne à la Brangerie 2 et 3. In : PUEYO (C.) dir., Villognon, Charente. LGV SEA - Villognon. La Brangerie, Rapport final d'opération de fouille, Inrap GSO-Inrap LGV SEA, L'Isle-d'Espagnac, 2013, p. 163-257.

Bats, Pueyo 2011 : BATS (J.-C.), PUEYO (C.) - Villognon, Charente. LGV SEA 2 Phase 52. Zone de dépôt de matériaux. DED 1 et 2, Rapport final d'opération de diagnostic, Inrap GSO-Inrap LGV SEA, L'Isle-d'Espagnac, 2011, 90 p., 45 fig.

Bertrand $\boldsymbol{e t} \boldsymbol{a l} .2009$ : BERTRAND (I.), DUVAL (A.), GOMEZ DE SOTO (J.), MAGUER (P.) dir., Habitats et paysages ruraux en Gaule et regards sur d'autres régions du monde celtique. Actes du XXXI colloque international de l'AFEAF (Chauvigny, 17-20 mai 2007). Chauvigny, Association des Publications Chauvinoises, 2009, 541 p. (Mémoire APC, XXXV).

Brun, Marcigny 2012 : BRUN (P.), MARCIGNY (C.) - Une connaissance de l'âge du Bronze transfigurée par l'archéologie préventive, Archéopages, hors série, janvier 2012, p. 133-139.

Buchsenschutz 2009 : BUCHSENSCHUTZ (O.) - Chars, charrettes et transport dans l'agriculture celtique. In : BERTRAND (I.), DUVAL (A.), GOMEZ DE SOTO (J.), MAGUER (P.) dir., Habitats et paysages ruraux en Gaule et regards sur d'autres régions du monde celtique. Actes du XXXI colloque international de l'AFEAF (Chauvigny, 17-20 mai 2007), Tome II. Chauvigny, Association des Publications Chauvinoises, 2009, p. 85-92 (Mémoire APC, XXXV).

Desbrosses, Riquier 2012 : DESBROSSES (V.), RIQUIER (V.) - Les établissements ruraux palissadés hallstattiens en Champagne. In : SCHÖNFELDER (M.), SIEVERS (S.) dir., L'âge du Fer entre la Champagne et la vallée du Rhin. Actes du XXXIV colloque international de l'AFEAF (Aschaffenburg, 13-16 mai 2010). Mainz, Verlag des Römisch-Germanischen Zentralmuseums, 2012, p. 3-27.
Fouéré et al. 2011 : FOUÉRÉ (P.) dir., avec la coll. ALLENET DE RIBEMONT (G.), DUSSOT (D.), GÉ (T.), GUITTON (D.), KEROUANTON (I.), MARTIN (H.), PELLISSIER (J.), POIRIER (P.), RAUX (S.), ROGER (J.), ROUSSEAU (J.), SIMON-HIERNARD (D.) - Regards sur l'occupation du plateau des Varennes depuis le Néolithique jusqu'à l'Antiquité, Rapport final d'opération de fouille, Inrap GSO, Poitiers, 2011, 3 vol.

Kerouanton et al. sous presse : KEROUANTON (I.), MAITAY (C.), BEAUSOLEIL (J.-M.) - L'habitat et l'occupation du sol à l'âge du Bronze dans le Centre-Ouest de la France (Poitou, Charentes et limousin). In : CAROZZA(L.) MARCIGNY (C.), TALON (M.) dir., L'habitat et l'occupation du sol à l'âge du Bronze et au premier âge du Fer : restitution de l'enquête nationale. Actes de la table ronde de Bayeux (29-30 novembre 2011). Paris, Éditions InrapCNRS, sous presse.

Landreau et al. 2009 : LANDREAU (G.), avec la coll. de ZÉLIE (B.), BARDOT (X.), HOUDUSSE (B.), MARATIER (B.), HESS (S.), ROUSSEAU (J.) Entre Isthme gaulois et Océan, la Saintonge au second âge du Fer. État des connaissances. In : BERTRAND (I.), DUVAL (A.), GOMEZ DE SOTO (J.), MAGUER (P.) dir., Les Gaulois entre Loire et Dordogne. Actes du XXXI ${ }^{\mathrm{e}}$ colloque international de l'AFEAF (Chauvigny, 17-20 mai 2007), Tome I. Chauvigny, Association des Publications Chauvinoises, 2009, p. 245-306 (Mémoire $A P C$, XXXIV)

Lavoix et al. 2013 : LAVOIX (G.), AUDÉ (V.), GERBER (F.), MARTINS (D.) dir., avec la coll. de ARD (V.), ARQUÉ (G.), BAUDRY (A.), CONNET (N.), DIETSCH-SELLAMI (M.-F.), FARAGO (B.), GEORGES (K.), GUITTON (D.), KEROUANTON (I.), LINLAUD (M.), MAITAY (C.), POIRIER (P.), VÉQUAUD (B.), VERDIN (P.) - 3500 ans d'occupation rurale à Jaunay-Clan. La Viaube 1 à Jaunay-Clan, Vienne, Rapport final d'opération de fouille, Inrap GSO, Poitiers, 2013, 3 vol.

Le Clézio, Giovannacci 2014 : LE CLÉZIO (L.), GIOVANNACCI (S.) - Un habitat ouvert en fond de vallée daté du premier âge du Fer « ZAE des Terres Rouges - Lot $3 »$ à Ingrandes (Vienne, Poitou-Charentes), Aquitania, 30 (2015), 2014, p. 47-60.

Lejars et al. 1990 : LEJARS (T.), LAPORTE (L.), PÉTORIN (N.) - Le Pédeau, site d'habitat protohistorique. Préguillac, Charente-Maritime. Fondation Électricité de France, 1990, n. p.

Maguer, Pautreau 2007 : MAGUER (P.), PAUTREAU (J.-P.) - L'ensemble céramique de La Tène ancienne du site d'habitat de Terre-qui-Fume à Buxerolles, Vienne. In : DUVAL (A.), GOMEZ DE SOTO (J.) dir., Sites et mobiliers de l'Âge du Fer entre Loire et Dordogne. Chauvigny, Association des Publications Chauvinoises, 2007, p. 86-90 (Mémoire APC, XXIX).

Maguer, Lusson 2009 : MAGUER (P.), LUSSON (D.), avec la coll. de TROUBADY (M.) - Fermes, hameaux et résidences aristocratiques entre Loire et Dordogne. In : BERTRAND (I.), DUVAL (A.), GOMEZ DE SOTO (J.), MAGUER (P.) dir., Les Gaulois entre Loire et Dordogne. Actes du XXXI ${ }^{e}$ colloque international de l'AFEAF (Chauvigny, 17-20 mai 2007), Tome I. Chauvigny, Association des Publications Chauvinoises, 2009, p. 423-459 (Mémoire APC, XXXIV).

Maguer et al. 2009 : MAGUER (P.), LANDREAU (G.), DUPONT (C.), MARTIN (H.), BARDOT (X.), POUPONNOT (G.), BRIAND (D.), DUVAL (A.) - L'habitat littoral des Ormeaux à Angoulins (Charente-Maritime). Activités vivrières et salicoles 
Références bibliographiques

entre marais et océan. In : BERTRAND (I.), DUVAL (A.), GOMEZ DE SOTO (J.), MAGUER (P.) dir., Les Gaulois entre Loire et Dordogne. Actes du XXXI' colloque international de l'AFEAF (Chauvigny, 17-20 mai 2007), Tome I. Chauvigny, Association des Publications Chauvinoises, 2009, p. 57-102 (Mémoire APC, XXXIV).

Maguer et al. 2010 : MAGUER (P.) dir., avec la coll. de BAMBAGIONI (F.), BILLY (B.), BERNARD (R.), COUTUREAU (M.), GEORGES (K.), GUITTON (D.), MAITAY (C.), VÉQUAUD (B.) - 6000 ans d'occupation humaine en bord de Vienne, au lieu-dit les Terres Rouges. Ingrandes-sur-Vienne, Vienne, Rapport final d'opération de diagnostic, Inrap GSO, Poitiers, 2010, 125 p., 78 fig.

Maguer, Robert 2013 : MAGUER (P.), ROBERT (G.) - La maison gauloise dans l'ouest et le centre de la France. In : KRAUSZ (S.) et al. dir., L'âge du Fer en Europe. Mélanges offerts à Olivier Buchsenschutz. Bordeaux, Ausonius Éditions, Mémoire 32, 2013, p. 247-258.

Maguer et al. 2014 : MAGUER (P.) dir., avec la coll. de ARD (V.), BAUDRY (A.), BERTRAND (I.), GENEVIĖVE (V.), GUITTON (D.), LEMAÎTRE (S.), LINLAUD (M.), MAITAY (C.), MOUGNE (C.), PICHON (M.), POIRIER (P.) - L'établissement rural gaulois des Gains à Saint-Georges-Lès-Baillargeaux (Vienne) : entre tradition et acculturation, Rapport final d'opération de fouille, Inrap GSO, Poitiers, 2014, 3 vol.

Maitay et al. 2009a : MAITAY (C.), MARCHADIER (É.), avec la coll. de BÉHAGUE (B.) - Entre traditions locales et apports exogènes : évolution et singularités du mobilier céramique du premier âge du Fer entre Loire et Dordogne. In : BERTRAND (I.), DUVAL (A.), GOMEZ DE SOTO (J.), MAGUER (P.) dir., Les Gaulois entre Loire et Dordogne. Actes du XXXI colloque international de l'AFEAF (Chauvigny, 17-20 mai 2007), Tome I. Chauvigny, Association des Publications Chauvinoises, 2009, p. 307-340 (Mémoire APC, XXXIV).

Maitay et al. 2009b : MAITAY (C.) coord., BÉHAGUE (B.), COLIN (A.), DUCONGÉ (S.), GOMEZ de SOTO (J.), KEROUANTON (I.), LANDREAU (G.), LARUAZ (J.-M.), LEVILLAYER (A.), ROUZEAU (N.), SIREIX (C.), SOYER (C.), VUAILLAT (D.), ZÉLIE (B.) - Forme et variabilité des habitats fortifiés des âges du Fer dans le Centre-Ouest de la France et ses marges. In : BERTRAND (I.), DUVAL (A.), GOMEZ DE SOTO (J.), MAGUER (P.) dir., Les Gaulois entre Loire et Dordogne. Actes du XXXI colloque international de l'AFEAF (Chauvigny, 17-20 mai 2007), Tome I. Chauvigny, Association des Publications Chauvinoises, 2009, p. 371-421 (Mémoire APC, XXXIV).

Maitay 2012 : MAITAY (C.) - Un exemple d'occupation rurale du premier âge du Fer dans le Centre-Ouest de la France. La ferme des Drouillards à Dompierresur-Mer, Charente-Maritime, Bulletin de l'Association des archéologues de Poitou-Charentes, no 41, 2012, p. 9-17.

Maitay et al. 2012 : MAITAY (C.) dir., avec la coll. de GEORGES (K.), POIRIER (P.) - Les Grands Philambins. Une occupation rurale de la fin de l'âge du Bronze sur la rive gauche de la vallée du Clain, Rapport final d'opération de fouille, Inrap GSO, Poitiers, 2012, 128 p., 76 fig.

Maitay en préparation : MAITAY (C.) - L'occupation rurale de l'âge du Bronze ancien et final de la Viaube 2 à Jaunay-Clan, Vienne, Rapport final d'opération de fouille, Inrap GSO, Poitiers, en préparation.

Maitay, Nillesse 2014 : MAITAY (C.), NILLESSE (O.) coord. - Occupation du sol et cultures matérielles au premier âge du Fer dans l'ouest de la Gaule. Actualités du premier âge du Fer dans l'ouest de la France. Publication du Séminaire archéologique de l'ouest, organisé au musée Sainte-Croix de Poitiers (18 octobre 2012), Aquitania, 30 (2015), 2014, p. 7-167;
Malrain, Blancquaert 2009 : MALRAIN (F.), BLANCQUAERT (G.) avec la coll. de LORHO (T.) - Un enclos $=$ une ferme ? In : BERTRAND (I.), DUVAL (A.), GOMEZ DE SOTO (J.), MAGUER (P.) dir., Les Gaulois entre Loire et Dordogne. Actes du XXXI colloque international de l'AFEAF (Chauvigny, 1720 mai 2007), Tome II. Chauvigny, Association des Publications Chauvinoises, 2009, p. 25-43 (Mémoire APC, XXXV).

Marcigny, Ghesquière 2003 : MARCIGNY (C.), GHESQUIÈRE (E.), avec la coll. de GALLOUIN (É.), GAUMÉ (É.), GIAZZON (D.) - Parcellaires et nécropoles de l'Âge du Bronze ancien à Bernières-sur-Mer (Calvados), BSPF, 100, 1, 2003, p. 117-134.

Marcigny et al. 2007 : MARCIGNY (C.), GHESQUIÈRE (E.), LESPEZ (L.), CLET-PELLERIN (M.) - Emprise ou déprise agricole à l'âge du Bronze moyen sur le littoral de la Manche ? Une lecture du phénomène grâce aux sites normands. In : Environnements et cultures à l'âge du Bronze en Europe occidentale. Actes du $129^{\mathrm{e}}$ congrès national des sociétés historiques et scientifiques (Besançon, 2004). CTHS, Collection Documents préhistoriques 21, 2007, p. 311-326.

Marcigny 2012 : MARCIGNY (C.) - Lieux funéraires, paysages et territoires de l'âge du Bronze en Normandie. In : Paysages funéraires de l'âge du Bronze. Actes du colloque international sur l'âge du Bronze (Herne, 15-18 octobre 2008). Darmstadt, Verlag Philipp von Zabern, 2012, p. 595-617.

Pautreau 1981 : PAUTREAU (J.-P.) - L'habitat protohistorique du Coteau de Montigné à Coulon (Deux-Sèvres). Travaux 1979-1980, Bulletin de la Société historique et scientifique des Deux-Sèvres, $2^{\mathrm{e}}$ série, XIII, 2-3, 1981, p. 95-137

Pautreau 1983 : PAUTREAU (J.-P.) - L'habitat protohistorique du Coteau de Montigné à Coulon (Deux-Sèvres). Travaux 1981-1982, Bulletin de la Société historique et scientifique des Deux-Sèvres, Actes du Congrès de Bressuire, $2^{\mathrm{e}}$ série, XV, 2-3, 1983, p. 101-149.

Pautreau, Gomez de Soto 1999 : PAUTREAU (J.-P.), GOMEZ DE SOTO (J.) - Les structures de stockage de l'âge du Fer dans le Centre-Ouest de la France : un bilan, Serie Monographica del MAC-Girona, 18, 1999, p. 333-338.

Pueyo et al. 2013 : PUEYO (C.), BARDOT (X.), DIOT (É.) dir., avec la coll. de ARRIGHI (V.), AUDÉ (V.), BERNARD (R.), BIDART (P.), BILLON (D.), BRISACH (B.), COUTUREAU (M.), DIETSCH-SELLAMI (M.-F.), GALLAIS (T.), GUIX (M.), MAITAY (C.), MARTIN (S.), MARTIN (W.), NEURY (P.), POIRIER (P.), SELLAMI (F.) - Villognon, Charente. LGV SEA - Villognon. La Brangerie, Rapport final d'opération de fouille, Inrap GSO-Inrap LGV SEA, L'Isle-d'Espagnac, 2013, 2 vol.

Sartou, Delalande $\boldsymbol{e} \boldsymbol{t} \boldsymbol{a l} .2012$ : SARTOU (A.), DELALANDE (A.) dir., avec la coll. de BERTHON (A.-A.), BIDAULT (E.), CURE (L.), DUCLOS (É.), FERDINAND (L.), LE CLÉZIO (L.), LEROY (B.), MACOUIN (M.), ROBIN (B.), TASTAVIN (A.) - Malaguet, Migné-Auxances (Vienne). LGV SEA 2 - ToursBordeaux, Rapport final d'opération, Éveha, 2012, 3 vol.

Séjalon et al. 2009 : SÉJALON (P.), BEL (V.), BREUIL (J.-Y.), POMARÈDES (H.) - Définition et organisation des terroirs protohistoriques de Nîmes, Gard (de la fin du VI ${ }^{\mathrm{e}}$ au $\mathrm{I}^{\mathrm{er}} \mathrm{s}$. av. J.-C.). In : BERTRAND (I.), DUVAL (A.), GOMEZ DE SOTO (J.), MAGUER (P.) dir., Les Gaulois entre Loire et Dordogne. Actes du XXXI ${ }^{e}$ colloque international de l'AFEAF (Chauvigny, 17-20 mai 2007), Tome II. Chauvigny, Association des Publications Chauvinoises, 2009, p. $153-$ 180 (Mémoire APC, XXXV). 
